\title{
杂芳基烷基醚、杂芳基卤化物与(氞代)醇的亲核醚化
}

\author{
王 霞 ${ }^{\dagger}$ 屈益欣 ${ }^{\dagger}$ 龙城宇 王雪强* \\ (湖南大学化学化工学院 分子科学与生物医学实验室(MBL) 化学/生物传感与化学计量学 \\ 国家重点实验室 长沙 410082)
}

\begin{abstract}
摘要 研究了在叔丁醇钾的作用下, 杂芳基烷氧化合物、杂芳基卤化物和杂芳基硫醚与各种伯、仲、叔脂肪醇高效梄 化, 强调了天然产物和生物活性分子的衍生化以及不易通过其他途径获得的気代芳基烷基醚的合成方法的实用性.

关键词＼cjkstart醚化; 杂芳基烷基梄; 芳香亲核取代
\end{abstract}

\section{Nucleophilic Etherification of Heteroaryl Alkyl Ethers, Heteroaryl Halides with (Deuterated) Alcohols}

\author{
Wang, Xia ${ }^{\dagger} \quad \mathrm{Qu}$, Yixin $^{\dagger} \quad$ Long, Chengyu Wang, Xue-Qiang* \\ (Molecular Science and Biomedicine Laboratory, State Key Laboratory of Chemo/Bio-sensing and Chemometrics, \\ College of Chemistry and Chemical Engineering, Hunan University, Changsha 410082)
}

\begin{abstract}
A KOt-Bu empowered transition-metal free and efficient procedure for the etherification of alkoxy heteroarenes, heteroaryl halides and heteroaryl thioethers with various primary, secondary, tertiary aliphatic alcohols has been developed. The usefulness of our method is highlighted by the derivatization of natural products and bioactive molecules, and the synthesis of deuterated heteroaryl alkyl ethers that are not easy to access via other approaches.

Keywords etherification; heteroaryl alkyl ethers; nucleophilic aromatic substitution
\end{abstract}

\section{Introduction}

$N$-Heteroaryl ethers are pervasive structural motifs existing in numerous natural products and top-selling pharmaceuticals on the market today, ${ }^{[1]}$ such as anticancer drugs crizotinib and olmutinib (Figure 1, Part I). ${ }^{[2]}$ In the past few decades, transition-metal-catalyzed etherification between readily available heteroaryl halides and abundant alcohols has become an efficient and fundamental technique in both academic laboratories and industrial manufacture. ${ }^{[3]}$ It can be attributed to its remarkable merits like broad substrate scope, mild reaction conditions, and the ability of complex settings (Figure 1, Part II). For instance, Buchwald and co-workers ${ }^{[4]}$ developed a Pd-catalyzed intermolecular $\mathrm{C}-\mathrm{O}$ bond formation promoted by a single phosphine ligand. Earlier this year, Ma group ${ }^{[5]}$ reported a general and mild $\mathrm{Cu}$ /oxalic diamides-catalyzed etherification of (hetero)aryl halides and alcohols to synthesize a wide number of complicated ethers. Notably, the photoredox catalysis strategy could also be utilized to prepare het- eroaryl ethers. MacMillan and his colleagues ${ }^{[6]}$ developed a highly efficient and general $\mathrm{C}-\mathrm{O}$ coupling reaction by merging Ni- and Ir-catalysts. Besides, transition-metal free methods such as Williamson ether synthesis, ${ }^{[7]}$ Mitsnobu reaction, ${ }^{[8]}$ and nucleophilic aromatic substitution are reliable alternatives to access $N$-heteroaryl ethers. ${ }^{[9]}$ Nevertheless, these methods suffer from several drawbacks, such as the use of toxic agents, expensive chemicals, toxic phosphines, and highly activate aryl fluorides, thus significantly hampering their industrial applications. Despite the tremendous advances in $\mathrm{C}-\mathrm{O}$ bond formation have been achieved, ${ }^{[10]}$ the etherification of methoxy heteroarenes via $\mathrm{C}-\mathrm{O}$ bond cleavage is remained limited success either using transition-metal catalysis or under metal-free reaction conditions probably due to the high dissociation energy of $\mathrm{C}\left(\mathrm{sp}^{2}\right)$ - OMe bond.

Our design of a direct etherification of methoxy pyridines was based on the mechanism of a classic reaction of pyridyl fluorides, nucleophilic aromatic substitution. ${ }^{[11]}$

\footnotetext{
* Corresponding author. E-mail: wangxq@hnu.edu.cn

Received June 30, 2020; revised August 20, 2020; published online September 10, 2020.

Project supported by the Huxiang Young Talent Program from Hunan Province (No. 2019RS2022).

湖南省湖湘青年人才计划(No. 2019RS2022)资助项目.

共同第一作者(These authors contributed equally to this work).
} 
Part I: Anticancer drugs containing $N$-heteroaryl ether

Crizotinib c-Met and ALK inhibitor

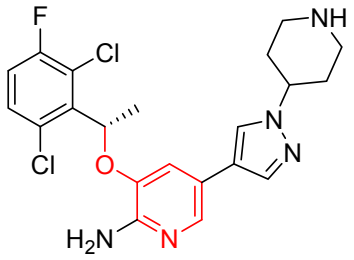

Olmutinib

EGFR tyrosine kinase inhibitor

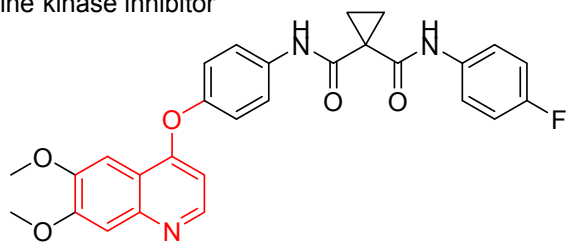

Part II: Etherification of N-heteroaromatics

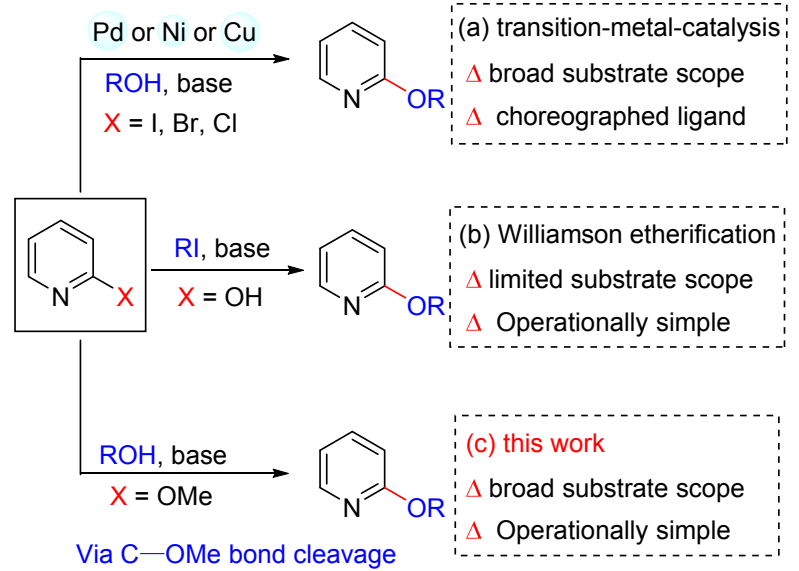

Figure 1 Representative ether containing anticancer drugs and the methods for heteroaryl ether synthesis.

This reaction starts with the nucleophilic attack of various nucleophiles to form Meisenheimer intermediate, followed by $\mathrm{C}-\mathrm{F}$ cleavage to give final products. We envisioned that an etherification of methoxy pyridines could be developed via nucleophilic alkoxy interconversion with alcohol if appropriate reaction conditions could be established after careful optimization (Figure 1, Part II).

\section{Results and discussion}

Promoted by our hypothesis, we started our investigation by study the reactivity of 2-methoxy pyridine (1a) with menthol. To our delight, the optimized reaction conditions was obtained after a detailed screening. It was found that the desired product 2a was generated in 95\% isolated yield under the optimized reaction conditions: potassium tert-butoxide ( $\mathrm{KO}$ - $\mathrm{Bu}, 2.0$ equiv.) in 1,4-dioxane $\left(1 \mathrm{~mol} \cdot \mathrm{L}^{-1}\right)$ at $100{ }^{\circ} \mathrm{C}$ for $16 \mathrm{~h}$ (Table $\left.1,1 \mathrm{a}\right)$. To investigate whether this reaction proceeds via the trace amount of transition metal within $\mathrm{KO} t-\mathrm{Bu}$, the etherification of 2-methoxypyridine with menthol was set up in the presence of $\mathrm{KO} t$-Bu with $99.99 \%$ purity, and the desired product was produced in $92 \%$ yield. It was indicated that this reaction might not occur via transition-metal catalysis pathway. With these encouraging results in hand, our attention to investigate the other alkoxy pyridines such as $\mathbf{1 b}$ and 1c was next turned. As expected, they could proceed with the etherification smoothly to produce the aimed ethers. The decreased yields might be caused by the increased steric requirement of ethoxy and butoxy groups. In addition, methylthio group could serve as an excellent leaving group, and $92 \%$ isolated yield was obtained while 2-(methylthio)pyrazine (1d) was subjected to the standard reaction conditions. Noteworthy, "unusual" starting materials for instance bromopyridines (1e, 1f) and iodopyridines $(\mathbf{1 g}, \mathbf{1 h})$ could also take part in the nucleophilic aromatic substitution reactions, moderate to good yields were observed. These results excellently highlighted the usefulness of our newly established mothed for $N$-heteroaryl alkyl ether construction. With these results in hand, we next focused on the representative scope of this etherification protocol, and the results were summarized in Table 2. The etherification with menthol proceeded smoothly at the $p a$ $r a$-position of methoxy pyridine, and $4 \mathbf{a}$ was offered in $81 \%$ yield. It is worth pointing out that this reaction could be scaled up to $10 \mathrm{mmol}$, generating the final ether in $87 \%$ yield $(1.891 \mathrm{~g})$. Chloro- at meta-position was well-tolerated with the standard conditions, $\mathbf{4 b}$ and $\mathbf{4 c}$ were isolated in excellent yields. Excellent regioselectivity was observed while 2,3-dimethoxy substituted pyridine was employed as starting material, alcohol interconversion only took place at ortho-position of pyridine while 3-methoxy was unreactive at all (4d). Notably, protected aldehyde was suited to go through etherification, thus leaving $4 \mathbf{e}$ as a useful synthon in organic synthetic chemistry. The etherification could be nicely extended to the other methoxy heteroarenes, such as pyrazine $(\mathbf{4 f})$, quinoline $(\mathbf{4 j})$, and pyrimidine (4k). Remarkably, double etherification was observed while two methoxy groups were located at the ortho-positions of pyridine and pyrimidine $(\mathbf{4 i}, \mathbf{4 k})$. The substrate scope of alcohol was also studied. Primary alcohols such as 4-phen- yl butanol, and the chiral center containing

Table 1 Scope of nucleofuges ${ }^{a}$
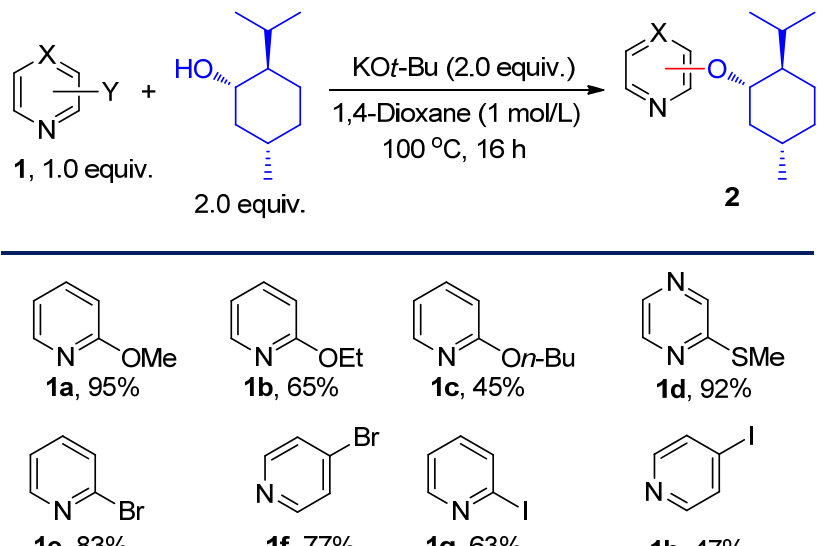

${ }^{a}$ Reaction conditions: azoheteroarene $(0.5 \mathrm{mmol})$, alcohol $(1.0 \mathrm{mmol}), \mathrm{KO} t-\mathrm{Bu}$ (1.0 mmol), 1,4-dioxane $\left(0.5 \mathrm{~mL}, 1 \mathrm{~mol} \cdot \mathrm{L}^{-1}\right), 100{ }^{\circ} \mathrm{C}, 16 \mathrm{~h}$. 
Table 2 Scope of methoxy heteroarenes and alcohols

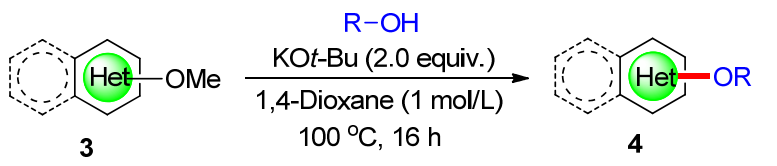

(a) scope of azoheteroarene

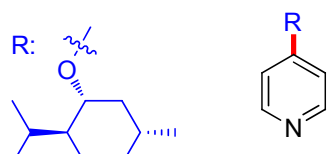

$4 a, 81 \%$

$4 a, 87 \%(1.891 \mathrm{~g})^{b}$<smiles>[R]c1ccc(Cl)cn1</smiles>

4b, $88 \%$<smiles>[R]c1ncccc1Cl</smiles>

4c, $85 \%$<smiles>[R]c1ncccc1OC</smiles><smiles>[R]c1cccc(C)n1</smiles><smiles>[R]c1cc(C)ccn1</smiles><smiles>CC(C)Oc1cccc(C2OCCO2)n1</smiles>

4d, $86 \%$

4e, $72 \%$

4f, $81 \%$

4g, $84 \%$<smiles>[R]c1cccc([R])n1</smiles>
4h, $64 \%$
$4 i, 60 \%{ }^{c}$
4j, $98 \%$
$4 \mathbf{k}, 64 \%^{c}$

(b) scope of alcohol<smiles>CCC(C)COc1ccncc1</smiles>

4I, $92 \%$<smiles>CCCCCCC(C)Oc1ccncc1</smiles><smiles>CC1(C)[C@H]2CC[C@@](C)(C2)[C@H]1Oc1ccncc1</smiles>

4n, $87 \%$<smiles>CN1C2CCC1C(Oc1ccccn1)C2</smiles><smiles>c1cc(O[C@@H]2CN3CC[C@H]2C3)ccn1</smiles>

4p, $87 \%$

$4 q, 88 \%$

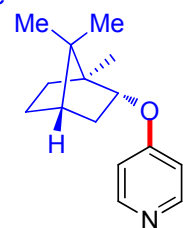

4r, $94 \%$<smiles>CCCCCCC(C)Oc1cc(C)nc2ccccc12</smiles>

4s, $73 \%$<smiles>CC(C)(C)Oc1cc(C(F)(F)F)nc2ccccc12</smiles>

4t, $39 \%$

${ }^{a}$ Reaction conditions: azoheteroarene $(0.5 \mathrm{mmol})$, alcohol $(1.0 \mathrm{mmol}), \mathrm{KO} t-\mathrm{Bu}$ (1.0 mmol), 1,4-dioxane $\left(0.5 \mathrm{~mL}, 1 \mathrm{~mol} \cdot \mathrm{L}^{-1}\right), 100{ }^{\circ} \mathrm{C}, 16 \mathrm{~h} .{ }^{b}$ Gram-scale reaction: 3a $(10 \mathrm{mmol})$, alcohol $(20 \mathrm{mmol}), \mathrm{KO} t$-Bu $(20 \mathrm{mmol})$, 1,4-dioxane $(50 \mathrm{~mL}), 100{ }^{\circ} \mathrm{C}, 16 \mathrm{~h} .{ }^{c} 3$ equivalents of alcohol and $\mathrm{KO} t$-Bu were used.

alcohol were suitable for this reaction, generating the corresponding products $4 \mathbf{I}$ and $\mathbf{4 m}$ in $92 \%$ and $68 \%$ yield, respectively. Similarly, simple secondary alcohols for instance 2-hep- tanol also exhibited high reactivity, obtaining the corresponding products $\mathbf{4 n}$ in $87 \%$ yield. In addition, a range of bio-related secondary alcohols like $(-)$-borneol (4o), $\beta$ - tropine (4p), $(R)$-quinuclidinol (4q), and ( + )-fenchol (4r) underwent alcohol interconversion without any problem, and the targeted products were produced in excellent yields. Apart from pyridines, methoxy quinoline

could take part in the etherification with good reactivity, reacted with secondary alcohol (4s) and even bulky tertiary alkoxide (4t) in good yields. ${ }^{[12]}$

We were also interested in utilizing this method to achieve late-stage derivatization of drugs or biorelevant molecules with 2-bromopyridine as modeling substrate. To prove this attribute, a range of drugs and bio-related molecules with structural and functional diversity (Table 3). Diosgenin, ${ }^{[13]}$ a STAT3 signaling pathway inhibitor incancer treatment, successfully reacted though in $87 \%$ yield (4u). Besides, the $\mathrm{C}-\mathrm{O}$ bond formation strategy was applied to the modification of diosgenin and cholesterol (es-

Table 3 Derivatization of pharmaceuticals or bio-related molecules

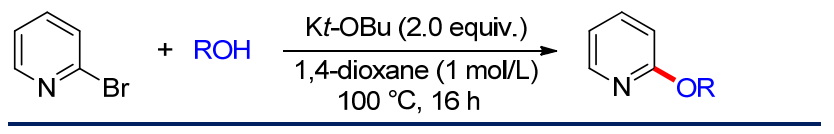

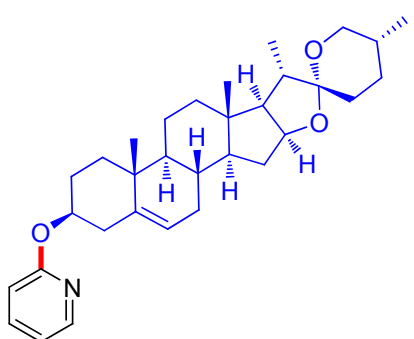

from diosgenin $4 u, 87 \%$

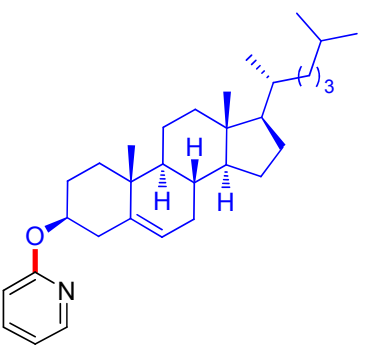

from cholesterol $4 \mathbf{v}, 42 \%$

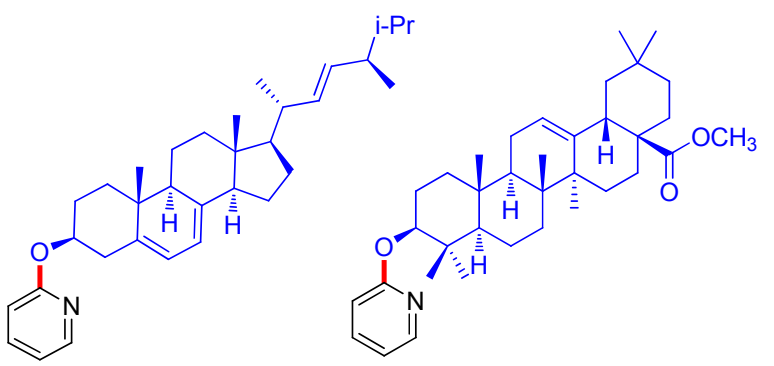

from ergosterol $\mathbf{4 w}, 71 \%$ from methyl oleanolate $4 \mathbf{x}, 52 \%$ from estradiol $\mathbf{4 y}, 98 \%$

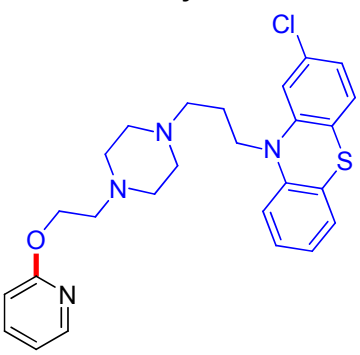

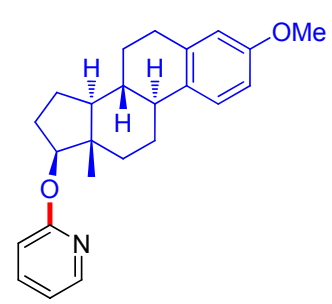

from perphenazine $\mathbf{4 a a}, 83 \%$

from abiraterone $\mathbf{4 z}, 81 \%$

${ }^{a}$ Reaction conditions: 1e $(0.2 \mathrm{mmol})$, alcohol $(0.4 \mathrm{mmol}), \mathrm{KO} t$-Bu $(0.4 \mathrm{mmol})$, 1,4-dioxane $\left(1.0 \mathrm{~mL}, 0.2 \mathrm{~mol} \cdot \mathrm{L}^{-1}\right), 100{ }^{\circ} \mathrm{C}, 16 \mathrm{~h}$. 
sential structural component of membranes), affording corresponding products $4 \mathbf{u}$ and $\mathbf{4 v}$ in $87 \%$ and $42 \%$ yields, respectively. It was also found that ergosterol and methyl oleanolate could react smoothly with $\mathbf{1 e}$, the desired products $4 \mathbf{w}$ and $4 x$ were obtained $71 \%$ and $52 \%$ yields, respectively. Excellent reactivity was observed as well when hormone type molecule such as estradiol derivative was subjected to the reaction system $(\mathbf{4 y})$. In addition, the use of an anticancer drug abiraterone generated the desired product in $81 \%$ yield (4z).$^{[14]}$ Antipsychotic drug perphenazine was also within reach under the standard reaction conditions, once again exhibiting the preparative potential of this protocol (4aa).$^{[15]}$

Next, the etherification reaction with deuterated alcohols was carried out as the introduction of deuterium into pharmaceuticals would greatly improve their pharmacodynamics and pharmacokinetic properties. ${ }^{[16]}$ First, the deuterated alcohols $\mathbf{S 1} \sim \mathbf{S 4}$ were synthesized by $\mathrm{NaBD}_{4}$ reducing corresponding aldehydes and ketones. As shown in Table 4, 2-methoxy substituted pyridines could efficiently react with deuterated hexanol derivative to give the corresponding ethers in good to excellent yields $(\mathbf{7 a} \sim \mathbf{7} \mathbf{c}$, 7e). Other heteroaryl methoxy ethers could participate in this alcohol interconversion reaction, $\mathbf{7 d}$ and $\mathbf{7 f}$ were respectively obtained in $72 \%$ and $91 \%$ isolated yields. The examination of the generality of alcohols demonstrated that other deuterated alcohols also have excellent reactivity $(7 \mathbf{g} \sim 7 \mathbf{j})$.

Next, the etherification reaction with deuterated alcohols was carried out as the introduction of deuterium into pharmaceuticals would greatly improve their pharmacodynamics and pharmacokinetic properties. ${ }^{[16]}$ First, the deuterated alcohols $\mathbf{S 1} \sim \mathbf{S} 4$ were synthesized by $\mathrm{NaBD}_{4}$ reducing corresponding aldehydes and ketones. As shown in Table 4, 2-methoxy substituted pyridines could efficiently react with deuterated hexanol derivative to give the corresponding ethers in good to excellent yields $(\mathbf{7} \mathbf{a} \sim \mathbf{7} \mathbf{c}$, 7e). Other heteroaryl methoxy ethers could participate in this alcohol interconversion reaction, $7 \mathbf{d}$ and $7 \mathbf{f}$ were respectively obtained in $72 \%$ and $91 \%$ isolated yields. The examination of the generality of alcohols demonstrated that other deuterated alcohols also have excellent reactivity $(7 \mathbf{g} \sim 7 \mathbf{j})$.

\section{Conclusions}

In summary, we have reported a base triggered practical yet environmentally benign etherification protocol that capable of efficiently incorporating primary, secondary, and even sterically bulky tertiary alcohols into azoheteroarenes via nucleophilic alkoxy interconversion, therefore generating pharmaceutical relevant ethers in good to excellent yields. This strategy could further be extended to the derivatization of a variety of drugs and biologically active molecules under standard reaction conditions. Similarly, a wide range of alcohols including primary and bulky secondary alcohols were suitable to undergo etherification.
Table 4 Scope of etherification of deuterated alcohols ${ }^{a}$

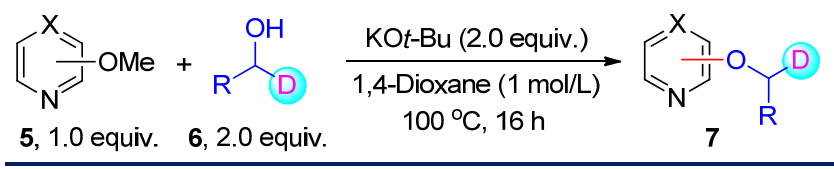

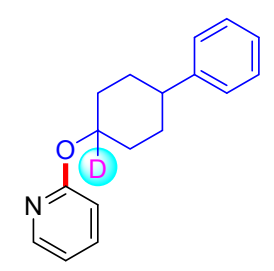

$7 a, 81 \%$
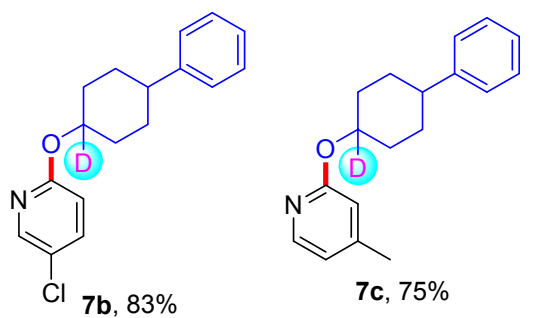

7c, $75 \%$

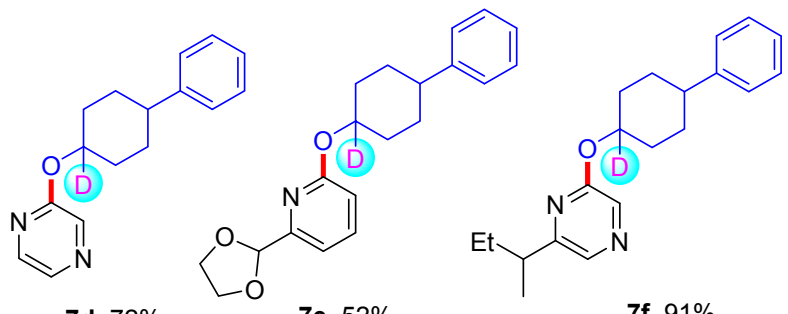

7d, $72 \%$

7f, $91 \%$

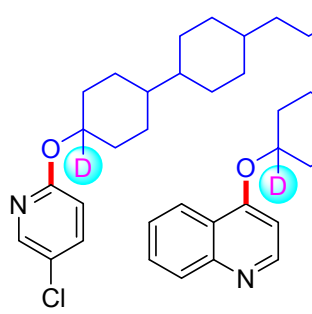

$7 \mathbf{g}, 86 \%$

7h, $69 \%$

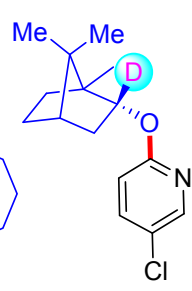

$7 \mathbf{i}, 96 \%$

7j, $91 \%$
${ }^{a}$ Reaction conditions: azoheteroarene $(0.5 \mathrm{mmol})$, deuterated alcohol $(1.0$ $\mathrm{mmol}), \mathrm{KO} t$-Bu $(1.0 \mathrm{mmol}), 1,4$-dioxane $\left(0.5 \mathrm{~mL}, 1 \mathrm{~mol} \cdot \mathrm{L}^{-1}\right), 100{ }^{\circ} \mathrm{C}, 16 \mathrm{~h}$.

\section{Experimental section}

\subsection{General methods}

All reactions were conducted in vials or Schlenck tubes under the nitrogen atmosphere. Chemical reagents were purchased from Energy Chemical, Bide Pharmatech Ltd and TCI, and used as received. The reactions were monitored by thin-layer chromatography (TLC), the products were purified by flash column chromatography, separately. ${ }^{1} \mathrm{H}$ NMR, and ${ }^{13} \mathrm{C}$ NMR spectra were included for all compounds. ${ }^{1} \mathrm{H}$ NMR and ${ }^{13} \mathrm{C}$ NMR spectra were recorded on a Bruker $400 \mathrm{MHz}$. All ${ }^{1} \mathrm{H}$ NMR spectra were measured relative to the signals for TMS $(\delta 0.00)$. All ${ }^{13} \mathrm{C}$ NMR spectra were reported relative to residual $\mathrm{DCCl}_{3}(\delta 77.0)$.

\subsection{General procedure}

Procedure I: To an oven-dried vial $(4 \mathrm{~mL})$ equipped with a magnetic stir bar, the vial was moved into a $\mathrm{N}_{2}$-filled glovebox. The vial was charged with heteroarene $(0.5$ $\mathrm{mmol}), \mathrm{KO} t-\mathrm{Bu}$ (1 $\mathrm{mmol})$, alcohol $(1 \mathrm{mmol})$, and 1,4-dioxane $(0.5 \mathrm{~mL})$. The vial was capped and the resulting reaction mixture was heated to $100{ }^{\circ} \mathrm{C}$ and stirred for $16 \mathrm{~h}$. The reaction mixture was then allowed to cool to room temperature, diluted with THF $(3 \mathrm{~mL})$, filtered through a plug of silica gel, and washed with THF. The 
crude mixture was concentrated in vacuo and purified by flash column chromatography on silica gel to provide the corresponding products $\mathbf{2 , 4}$ and 7 .

Procedure II: To an oven-dried vial $(4 \mathrm{~mL})$ equipped with a magnetic stir bar, the vial was moved into a $\mathrm{N}_{2}$ filled glovebox. The vial was charged with 2-bromopyridine $(0.2 \mathrm{mmol}), \mathrm{KO} t$ - $\mathrm{Bu}(0.4 \mathrm{mmol})$, alcohol $(0.4 \mathrm{mmol})$, and 1,4-dioxane $(1.0 \mathrm{~mL})$. The vial was capped, the resulting reaction mixture was heated $100{ }^{\circ} \mathrm{C}$ and stirred for 16 $\mathrm{h}$. The reaction mixture was then allowed to cool to room temperature, diluted with THF $(3 \mathrm{~mL})$, filtered through a plug of silica gel, and washed with THF. The crude mixture was concentrated in vacuo and purified by flash column chromatography on silica gel to provide the corresponding product 4.

Procedure III: A round bottom flask $(25 \mathrm{~mL})$ equipped with a magnetic stir bar was charged with methanol $(3 \mathrm{~mL})$ and $\mathrm{R}^{1} \mathrm{COR}^{2}$ ( $\left.1 \mathrm{mmol}\right)$ in ice bath, followed by adding $\mathrm{NaBD}_{4}(1.5 \mathrm{mmol})$ slowly, and covered with a rubber plug. The mixture was moved in room temperature and stirred for $2 \mathrm{~h}$. Then the reaction was quenched by water $(5 \mathrm{~mL})$ and extracted with ethyl acetate $(5 \mathrm{~mL} \times 3)$. The crude mixture was concentrated in vacuo and purified by flash column chromatography on silica gel to provide the corresponding products $\mathbf{S 1} \sim \mathbf{S 3}$.

2-(((1S,2R,5S)-2-Isopropyl-5-methylcyclohexyl)oxy)pyridine (2a): Following the general procedure I, using 1a, $\mathbf{1 b}, \mathbf{1 c}, \mathbf{1 e}$, and $\mathbf{1 g}$ as starting material to afford $\mathbf{2 a}$ in yields of $95 \%$ (110.6 mg), 65\% (75.8 mg), 45\% (52.4 mg), 83\% $(96.7 \mathrm{mg})$ and $63 \%(73.4 \mathrm{mg})$ as a colourless oil (eluent: $V($ hexane $/ V($ EtOAc $)=40 / 1) .{ }^{1} \mathrm{H}$ NMR $\left(400 \mathrm{MHz}, \mathrm{CDCl}_{3}\right)$ $\delta: 8.13(\mathrm{~d}, J=4.9 \mathrm{~Hz}, 1 \mathrm{H}), 7.52(\mathrm{t}, J=7.7 \mathrm{~Hz}, 1 \mathrm{H}), 6.79(\mathrm{t}$, $J=6.0 \mathrm{~Hz}, 1 \mathrm{H}), 6.66(\mathrm{~d}, J=8.3 \mathrm{~Hz}, 1 \mathrm{H}), 4.99$ (q, $J=10.7$, $4.2 \mathrm{~Hz}, 1 \mathrm{H}), 2.20(\mathrm{~d}, J=12.1 \mathrm{~Hz}, 1 \mathrm{H}), 2.06(\mathrm{q}, J=6.8 \mathrm{~Hz}$, $1 \mathrm{H}), 1.71(\mathrm{~d}, J=11.4 \mathrm{~Hz}, 2 \mathrm{H}), 1.52(\mathrm{q}, J=24.9,13.4 \mathrm{~Hz}$, $2 \mathrm{H}), 1.14$ (q, $J=13.0,12.3 \mathrm{~Hz}, 1 \mathrm{H}), 1.03 \sim 0.88(\mathrm{~m}, 8 \mathrm{H})$, $0.76(\mathrm{~d}, J=6.9 \mathrm{~Hz}, 3 \mathrm{H}) ;{ }^{13} \mathrm{C}$ NMR $\left(101 \mathrm{MHz}, \mathrm{CDCl}_{3}\right) \delta$ : 164.0, 147.0, 138.6, 116.2, 111.6, 74.6, 47.9, 41.0, 34.7, $31.5,26.5,23.9,22.3,20.9,16.8$; IR (neat) $v: 777,989$, 1290, 1430, 1590, $950 \mathrm{~cm}^{-1}$. HRMS (ESI) calcd for $\mathrm{C}_{15} \mathrm{H}_{24} \mathrm{NO}[\mathrm{M}+\mathrm{H}]^{+}: 234.1858$, found 234.1849.

4-(((1S,2R,5S)-2-Isopropyl-5-methylcyclohexyl)oxy)pyridine (4a): Following the general procedure $\mathbf{I}$, using 1f, $\mathbf{1 h}$, and $\mathbf{3 a}$ as starting material to afford $\mathbf{4 a}$ in yields of $77 \%(89.7 \mathrm{mg}), 47 \%(54.8 \mathrm{mg})$, and $81 \%(94.4 \mathrm{mg})$ as colourless oil (eluent: $V($ hexane $/ V($ EtOAc $)=10 / 1)$. m.p. $60.2{ }^{\circ} \mathrm{C} ;{ }^{1} \mathrm{H}$ NMR $\left(400 \mathrm{MHz}, \mathrm{CDCl}_{3}\right.$ ) $\delta: 8.38$ (s, 2H), 6.77 (s, 2H), $4.12(\mathrm{t}, J=10.5 \mathrm{~Hz}, 1 \mathrm{H}), 2.12(\mathrm{~d}, J=12.5 \mathrm{~Hz}, 2 \mathrm{H})$, $1.73(\mathrm{~d}, J=12.0 \mathrm{~Hz}, 2 \mathrm{H}), 1.64 \sim 1.41(\mathrm{~m}, 2 \mathrm{H}), 1.08(\mathrm{dt}$, $J=26.0,12.8 \mathrm{~Hz}, 2 \mathrm{H}), 0.91(\mathrm{~d}, J=7.6 \mathrm{~Hz}, 7 \mathrm{H}), 0.73$ (d, $J=5.3 \mathrm{~Hz}, 3 \mathrm{H}) ;{ }^{13} \mathrm{C} \mathrm{NMR}\left(101 \mathrm{MHz}, \mathrm{CDCl}_{3}\right) \delta: 164.5$, $151.2,110.9,77.2,47.8,39.8,34.4,31.5,26.2,23.8,22.2$, 20.7, 16.7; IR (neat) v: 95, 1210, 1280, 1500, 1590, 2930 $\mathrm{cm}^{-1}$. HRMS (ESI) calcd for $\mathrm{C}_{15} \mathrm{H}_{24} \mathrm{NO}[\mathrm{M}+\mathrm{H}]^{+}$: 234.1858, found 234.1847.

2-(((1S,2R,5S)-2-Isopropyl-5-methylcyclohexyl)oxy)pyrazine (2d): Following the general procedure $\mathbf{I}$ to afford $\mathbf{2 d}$
(107.6 mg, 92\% yield) as white solid (eluent: $V$ (hexane/ $V($ EtOAc $)=40 / 1)$. m.p. $59.5{ }^{\circ} \mathrm{C} ;{ }^{1} \mathrm{H}$ NMR $(400 \mathrm{MHz}$, $\left.\mathrm{CDCl}_{3}\right) \delta: 8.12(\mathrm{~s}, 1 \mathrm{H}), 8.01(\mathrm{~s}, 2 \mathrm{H}), 5.02 \sim 4.85(\mathrm{~m}, 1 \mathrm{H})$, $2.12(\mathrm{~d}, J=11.8 \mathrm{~Hz}, 1 \mathrm{H}), 2.06 \sim 1.90(\mathrm{~m}, 1 \mathrm{H}), 1.69(\mathrm{~d}, J=$ $11.8 \mathrm{~Hz}, 2 \mathrm{H}), 1.51(\mathrm{t}, J=10.7 \mathrm{~Hz}, 2 \mathrm{H}), 1.18 \sim 1.06(\mathrm{~m}$, $1 \mathrm{H}), 1.06 \sim 0.97(\mathrm{~m}, 1 \mathrm{H}), 0.90(\mathrm{~d}, J=14.8 \mathrm{~Hz}, 7 \mathrm{H}), 0.71$ $(\mathrm{d}, J=6.9 \mathrm{~Hz}, 3 \mathrm{H}) ;{ }^{13} \mathrm{C}$ NMR $\left(101 \mathrm{MHz}, \mathrm{CDCl}_{3}\right) \delta: 160.4$, 140.6, 136.5, 136.1, 75.6, 47.6, 40.5, 34.5, 31.5, 26.4, 23.8, 22.2, 20.9, 16.7; IR (neat) $v: 802,966,1210,1290,1410$, $1460,1530 \mathrm{~cm}^{-1}$. HRMS (ESI) calcd for $\mathrm{C}_{14} \mathrm{H}_{23} \mathrm{~N}_{2} \mathrm{O}[\mathrm{M}+$ $\mathrm{H}]^{+}:$235.1810, found 235.1803.

5-Chloro-2-(((1S,2R,5S)-2-isopropyl-5-methylcyclohexyl)oxy)pyridine (4b): Following the general procedure I to afford $\mathbf{4 b}(117.5 \mathrm{mg}, 88 \%$ yield) as colourless oil (eluent: $V($ hexane $/ V($ EtOAc $)=40 / 1) .{ }^{1} \mathrm{H}$ NMR $(400 \mathrm{MHz}$, $\left.\mathrm{CDCl}_{3}\right) \delta: 8.05(\mathrm{~s}, 1 \mathrm{H}), 7.46(\mathrm{~d}, J=8.8 \mathrm{~Hz}, 1 \mathrm{H}), 6.74 \sim$ $6.57(\mathrm{~m}, 1 \mathrm{H}), 4.93(\mathrm{t}, J=10.7 \mathrm{~Hz}, 1 \mathrm{H}), 2.15$ (d, $J=11.8$ $\mathrm{Hz}, 1 \mathrm{H}), 2.07 \sim 1.94(\mathrm{~m}, 1 \mathrm{H}), 1.69(\mathrm{~d}, J=11.1 \mathrm{~Hz}, 2 \mathrm{H})$, $1.50(\mathrm{dd}, J=23.8,11.4 \mathrm{~Hz}, 2 \mathrm{H}), 1.11$ (q, $J=12.8 \mathrm{~Hz}, 1 \mathrm{H})$, $0.92(\mathrm{~d}, J=27.6 \mathrm{~Hz}, 8 \mathrm{H}), 0.73(\mathrm{~d}, J=6.4 \mathrm{~Hz}, 3 \mathrm{H}) ;{ }^{13} \mathrm{C}$ NMR $\left(101 \mathrm{MHz}, \mathrm{CDCl}_{3}\right) \delta: 162.4,145.2,138.5,123.3$, $112.5,75.3,47.8,40.7,34.6,31.5,26.4,23.8,22.3,20.9$, 16.7; IR (neat) $v: 825,989,1280,1470,1910,2960 \mathrm{~cm}^{-1}$. HRMS (ESI) calcd for $\mathrm{C}_{15} \mathrm{H}_{22} \mathrm{ClNONa}[\mathrm{M}+\mathrm{Na}]^{+}$: 290.1288 , found 290.1283 .

3-Chloro-2-(( $1 S, 2 R, 5 S)$-2-isopropyl-5-methylcyclohexyl)oxy)pyridine (4c): Following the general procedure I to afford $4 \mathrm{c}(113.5 \mathrm{mg}, 85 \%$ yield) as colourless oil (eluent: $V($ hexane $/ V($ EtOAc $)=40 / 1) .{ }^{1} \mathrm{H}$ NMR $(400 \mathrm{MHz}$, $\left.\mathrm{CDCl}_{3}\right) \delta: 8.02(\mathrm{~s}, 1 \mathrm{H}), 7.60(\mathrm{~d}, J=7.4 \mathrm{~Hz}, 1 \mathrm{H}), 6.77(\mathrm{~s}$, $1 \mathrm{H}), 5.03$ (t, $J=10.6 \mathrm{~Hz}, 1 \mathrm{H}), 2.20$ (d, $J=11.8 \mathrm{~Hz}, 1 \mathrm{H})$, $2.16 \sim 2.00(\mathrm{~m}, 1 \mathrm{H}), 1.72(\mathrm{~d}, J=11.4 \mathrm{~Hz}, 2 \mathrm{H}), 1.61(\mathrm{~d}, J=$ $16.9 \mathrm{~Hz}, 2 \mathrm{H}), 1.11$ (dq, $J=22.5,12.1,11.5 \mathrm{~Hz}, 2 \mathrm{H}), 0.92$ $(\mathrm{d}, J=6.5 \mathrm{~Hz}, 7 \mathrm{H}), 0.75(\mathrm{~d}, J=6.2 \mathrm{~Hz}, 3 \mathrm{H}) ;{ }^{13} \mathrm{C} \mathrm{NMR}$ $\left(101 \mathrm{MHz}, \mathrm{CDCl}_{3}\right) \delta: 159.2,144.7,138.3,118.6,116.7$, $76.2,47.5,40.7,34.6,31.5,26.5,23.9,22.3,20.9,16.9$; IR (neat) $v: 793,1050,1320,1440,2960 \mathrm{~cm}^{-1}$. HRMS (ESI) calcd for $\mathrm{C}_{15} \mathrm{H}_{22} \mathrm{ClNONa}[\mathrm{M}+\mathrm{Na}]^{+}: 290.1288$, found 290.1283 .

2-(((1S,2R,5S)-2-Isopropyl-5-methylcyclohexyl)oxy)-3methoxypyridine (4d): Following the general procedure I to afford 4d (113.0 mg, 86\% yield) as white solid (eluent: $V($ hexane $/ V($ EtOAc $)=10 / 1)$. m.p. $80.2{ }^{\circ} \mathrm{C} ;{ }^{1} \mathrm{H}$ NMR $(400$ $\left.\mathrm{MHz}, \mathrm{CDCl}_{3}\right) \delta: 7.69(\mathrm{~s}, 1 \mathrm{H}), 7.00(\mathrm{~d}, J=7.5 \mathrm{~Hz}, 1 \mathrm{H})$, $6.76(\mathrm{~s}, 1 \mathrm{H}), 5.11(\mathrm{t}, J=10.5 \mathrm{~Hz}, 1 \mathrm{H}), 3.83(\mathrm{~s}, 3 \mathrm{H}), 2.19$ $(\mathrm{d}, J=11.7 \mathrm{~Hz}, 1 \mathrm{H}), 2.07(\mathrm{~s}, 1 \mathrm{H}), 1.80 \sim 1.50(\mathrm{~m}, 4 \mathrm{H})$, 1.12 (p, $J=11.1,10.7 \mathrm{~Hz}, 2 \mathrm{H}), 0.95 \sim 0.85(\mathrm{~m}, 7 \mathrm{H}), 0.74$ $(\mathrm{d}, J=6.4 \mathrm{~Hz}, 3 \mathrm{H}) ;{ }^{13} \mathrm{C} \mathrm{NMR}\left(101 \mathrm{MHz}, \mathrm{CDCl}_{3}\right) \delta: 154.3$, $144.4,137.1,117.5,116.1,74.9,55.8,47.5,40.9,34.7$, 31.6, 26.3, 23.8, 22.3, 20.9, 16.7; IR (neat) v: 987, 1120, $1210,1250,1460,1590,2950 \mathrm{~cm}^{-1}$. HRMS (ESI) calcd for $\mathrm{C}_{16} \mathrm{H}_{25} \mathrm{NO}_{2} \mathrm{Na}[\mathrm{M}+\mathrm{Na}]^{+}: 286.1783$, found 286.1770.

2-(((1S,2R,5S)-2-Isopropyl-5-methylcyclohexyl)oxy)-6methylpyridine (4e): Following the general procedure I to afford $4 \mathrm{e}$ (88.9 mg, 72\% yield) as colourless oil (eluent: $V($ hexane $/ V($ EtOAc $)=40 / 1) .{ }^{1} \mathrm{H}$ NMR $\left(400 \mathrm{MHz}, \mathrm{CDCl}_{3}\right)$ $\delta: 7.41(\mathrm{t}, J=7.5 \mathrm{~Hz}, 1 \mathrm{H}), 6.64(\mathrm{~d}, J=7.1 \mathrm{~Hz}, 1 \mathrm{H}), 6.46$ 
(d, $J=8.1 \mathrm{~Hz}, 1 \mathrm{H}), 4.98(\mathrm{t}, J=10.6 \mathrm{~Hz}, 1 \mathrm{H}), 2.41(\mathrm{~s}, 3 \mathrm{H})$, $2.17(\mathrm{~d}, J=11.9 \mathrm{~Hz}, 1 \mathrm{H}), 2.05(\mathrm{p}, J=6.7 \mathrm{~Hz}, 1 \mathrm{H}), 1.71$ (d, $J=10.4 \mathrm{~Hz}, 2 \mathrm{H}), 1.66 \sim 1.42(\mathrm{~m}, 2 \mathrm{H}), 1.13(\mathrm{q}, J=12.9 \mathrm{~Hz}$, $1 \mathrm{H}), 1.07 \sim 0.98(\mathrm{~m}, 1 \mathrm{H}), 0.91(\mathrm{t}, J=7.5 \mathrm{~Hz}, 7 \mathrm{H}), 0.77(\mathrm{~d}$, $J=6.6 \mathrm{~Hz}, 3 \mathrm{H}) ;{ }^{13} \mathrm{C} \mathrm{NMR}\left(101 \mathrm{MHz}, \mathrm{CDCl}_{3}\right) \delta: 163.4$, $156.4,138.7,115.2,107.4,74.2,48.1,41.0,34.7,31.6$, 26.3, 24.4, 23.7, 22.4, 21.0, 16.7; IR (neat) v: 793, 991, 1300, 1450, $2950 \mathrm{~cm}^{-1}$. HRMS (ESI) calcd for $\mathrm{C}_{16} \mathrm{H}_{25^{-}}$ $\mathrm{NONa}[\mathrm{M}+\mathrm{Na}]^{+}: 270.1834$, found 270.1828 .

2-(((1S,2R,5S)-2-Isopropyl-5-methylcyclohexyl)oxy)-4methylpyridine (4f): Following the general procedure I to afford 4f (100.0 mg, 81\% yield) as white solid (eluent: $V($ hexane $/ V($ EtOAc $)=40 / 1)$. m.p. $50{ }^{\circ} \mathrm{C} ;{ }^{1} \mathrm{H}$ NMR $(400$ $\left.\mathrm{MHz}, \mathrm{CDCl}_{3}\right) \delta: 8.01(\mathrm{~d}, J=4.7 \mathrm{~Hz}, 1 \mathrm{H}), 6.66(\mathrm{~s}, 1 \mathrm{H})$, $6.52(\mathrm{~s}, 1 \mathrm{H}), 4.99$ (t, $J=10.6 \mathrm{~Hz}, 1 \mathrm{H}), 2.29$ (s, 3H), 2.21 $(\mathrm{d}, J=11.9 \mathrm{~Hz}, 1 \mathrm{H}), 2.08(\mathrm{p}, J=6.7 \mathrm{~Hz}, 1 \mathrm{H}), 1.73(\mathrm{~d}, J=$ $10.6 \mathrm{~Hz}, 2 \mathrm{H}), 1.66 \sim 1.47(\mathrm{~m}, 2 \mathrm{H}), 1.16(\mathrm{q}, J=12.7 \mathrm{~Hz}$, $1 \mathrm{H}), 0.97$ (dd, $J=33.8,9.3 \mathrm{~Hz}, 8 \mathrm{H}), 0.78(\mathrm{~d}, J=6.7 \mathrm{~Hz}$, $3 \mathrm{H}) ;{ }^{13} \mathrm{C}$ NMR $\left(101 \mathrm{MHz}, \mathrm{CDCl}_{3}\right) \delta: 164.3,149.9,146.6$, 117.8, 111.6, 74.4, 48.0, 41.0, 34.7, 31.5, 26.5, 23.9, 22.3, 21.0, 20.9, 16.8; IR (neat) $v: 808,1030,1160,1320,1610$, $2950 \mathrm{~cm}^{-1}$. HRMS (ESI) calcd for $\mathrm{C}_{16} \mathrm{H}_{25} \mathrm{NONa}[\mathrm{M}+$ $\mathrm{Na}]^{+}: 270.1834$, found 270.1824 .

2-(1,3-Dioxolan-2-yl)-6-isopropoxypyridine (4g): Following the general procedure I to afford $\mathbf{4 g}(87.8 \mathrm{mg}, 84 \%$ yield) as colourless oil (eluent: $V($ hexane $/ V($ EtOAc $)=$ 40/1). ${ }^{1} \mathrm{H}$ NMR (400 MHz, $\left.\mathrm{CDCl}_{3}\right) \delta: 7.54$ (t, $J=7.7 \mathrm{~Hz}$, $1 \mathrm{H}), 7.02$ (d, $J=7.2 \mathrm{~Hz}, 1 \mathrm{H}), 6.63$ (d, $J=8.2 \mathrm{~Hz}, 1 \mathrm{H}), 5.75$ (s, 1H), 5.34 (p, J=6.0 Hz, 1H), 4.16 (s, 2H), 4.03 (s, 2H), $1.32(\mathrm{~d}, J=6.2 \mathrm{~Hz}, 6 \mathrm{H}) ;{ }^{13} \mathrm{C}$ NMR $\left(101 \mathrm{MHz}, \mathrm{CDCl}_{3}\right) \delta$ : 163.2, 154.8, 139.0, 112.6, 111.9, 103.7, 68.0, 65.5, 22.0; IR (neat) $v: 808,964,1110,1320,1450,1580,2980 \mathrm{~cm}^{-1}$. HRMS (ESI) calcd for $\mathrm{C}_{11} \mathrm{H}_{15} \mathrm{NO}_{3} \mathrm{Na}[\mathrm{M}+\mathrm{Na}]^{+}$: 232.0950, found 232.0943 .

2,6-Bis $(((1 S, 2 R, 5 S)$-2-Isopropyl-5-methylcyclohexyl)oxy)pyridine (4i): Following the general procedure $\mathbf{I}$ to afford 4i (116.1 mg, 60\% yield) as colourless oil (eluent: $V($ hexane $/ V($ EtOAc $)=40 / 1) .{ }^{1} \mathrm{H}$ NMR $\left(400 \mathrm{MHz}, \mathrm{CDCl}_{3}\right)$ $\delta: 7.51 \sim 7.36(\mathrm{~m}, 1 \mathrm{H}), 6.19(\mathrm{~d}, J=7.6 \mathrm{~Hz}, 2 \mathrm{H}), 4.92(\mathrm{t}$, $J=10.7 \mathrm{~Hz}, 2 \mathrm{H}), 2.24 \sim 2.01(\mathrm{~m}, 4 \mathrm{H}), 1.72(\mathrm{~d}, J=11.3 \mathrm{~Hz}$, $4 \mathrm{H}), 1.53$ (q, $J=26.8,12.4 \mathrm{~Hz}, 4 \mathrm{H}), 1.17 \sim 0.86(\mathrm{~m}, 18 \mathrm{H})$, $0.77(\mathrm{~d}, J=5.9 \mathrm{~Hz}, 6 \mathrm{H}) ;{ }^{13} \mathrm{C}$ NMR $\left(101 \mathrm{MHz}, \mathrm{CDCl}_{3}\right) \delta$ : $163.1,162.9,141.0,102.0,100.5,74.9,53.4,47.7,41.2$, $34.7,31.7,26.3,23.8,22.4,20.9,16.7$; IR (neat) $v$ : 793, 1030, 1240, 1440, 1600, $2950 \mathrm{~cm}^{-1}$. HRMS (ESI) calcd for $\mathrm{C}_{25} \mathrm{H}_{42} \mathrm{NO}_{2}[\mathrm{M}+\mathrm{H}]^{+}: 388.3216$, found 388.3201 .

4-(((1S,2R,5S)-2-Isopropyl-5-methylcyclohexyl)oxy)-2methylquinoline $(\mathbf{4 j})$ : Following the general procedure $I$ to afford $\mathbf{4 j}$ (145.5 mg, 98\% yield) as colourless oil (eluent: $V($ hexane $/ V($ EtOAc $)=5 / 1) .{ }^{1} \mathrm{H}$ NMR $\left(400 \mathrm{MHz}, \mathrm{CDCl}_{3}\right) \delta$ : $8.14(\mathrm{~d}, J=8.3 \mathrm{~Hz}, 1 \mathrm{H}), 7.93(\mathrm{~d}, J=8.4 \mathrm{~Hz}, 1 \mathrm{H}), 7.62$ (t, $J=7.6 \mathrm{~Hz}, 1 \mathrm{H}), 7.40$ (t, $J=7.6 \mathrm{~Hz}, 1 \mathrm{H}), 6.61$ (s, 1H), 4.33 $(\mathrm{q}, J=10.5,4.1 \mathrm{~Hz}, 1 \mathrm{H}), 2.69(\mathrm{~s}, 3 \mathrm{H}), 2.26(\mathrm{~d}, J=12.5 \mathrm{~Hz}$, $1 \mathrm{H}), 2.17(\mathrm{q}, J=13.6,5.9 \mathrm{~Hz}, 1 \mathrm{H}), 1.84 \sim 1.66(\mathrm{~m}, 3 \mathrm{H})$, $1.57(\mathrm{~s}, 1 \mathrm{H}), 1.20 \sim 1.06(\mathrm{~m}, 2 \mathrm{H}), 1.06 \sim 0.90(\mathrm{~m}, 7 \mathrm{H})$, $0.76(\mathrm{~d}, J=6.9 \mathrm{~Hz}, 3 \mathrm{H}) ;{ }^{13} \mathrm{C} \mathrm{NMR}\left(101 \mathrm{MHz}, \mathrm{CDCl}_{3}\right) \delta$ : $161.0,160.1,149.2,129.7,128.1,124.6,122.0,120.6$,
$101.2,77.8,48.0,39.6,34.5,31.5,26.6,26.1,24.0,22.2$, 20.8, 17.0; IR (neat) $v: 764,984,1110,1420,1590,2950$ $\mathrm{cm}^{-1}$. HRMS (ESI) calcd for $\mathrm{C}_{20} \mathrm{H}_{28} \mathrm{NO}[\mathrm{M}+\mathrm{H}]^{+}$: 298.2171, found 298.2158.

4-(((1R,2R,5S)-2-Isopropyl-5-methylcyclohexyl)oxy)-2(((1S,2R,5S)-2-isopropyl-5-methylcyclohexyl)oxy)pyrimidine (4k): Following the general procedure $I$ to afford $4 \mathbf{k}$ (124.2 mg, 64\% yield) as colourless oil (eluent: $V$ (hexane/ $V($ EtOAc $)=20 / 1) .{ }^{1} \mathrm{H}$ NMR $\left(400 \mathrm{MHz}, \mathrm{CDCl}_{3}\right) \delta: 8.24 \sim$ $8.09(\mathrm{~m}, 1 \mathrm{H}), 6.25(\mathrm{~d}, J=3.4 \mathrm{~Hz}, 1 \mathrm{H}), 5.14(\mathrm{t}, J=10.7 \mathrm{~Hz}$, $1 \mathrm{H}), 4.94(\mathrm{t}, J=10.7 \mathrm{~Hz}, 1 \mathrm{H}), 2.13(\mathrm{t}, J=12.1 \mathrm{~Hz}, 3 \mathrm{H})$, $2.03 \sim 1.88(\mathrm{~m}, 1 \mathrm{H}), 1.71(\mathrm{~d}, J=11.3 \mathrm{~Hz}, 4 \mathrm{H}), 1.56(\mathrm{q}, J=$ 44.4, $11.2 \mathrm{~Hz}, 4 \mathrm{H}), 1.10$ (q, $J=19.1,10.7 \mathrm{~Hz}, 4 \mathrm{H}), 0.90$ (d, $J=6.3 \mathrm{~Hz}, 14 \mathrm{H}), 0.77(\mathrm{~d}, J=6.4 \mathrm{~Hz}, 6 \mathrm{H}) ;{ }^{13} \mathrm{C} \mathrm{NMR}(101$ $\left.\mathrm{MHz} \mathrm{CDCl}_{3}\right) \delta: 171.1,165.1,158.7,102.0,76.7,75.4$, $47.6,47.1,40.7,40.7,34.5,34.4,31.7,31.5,26.4,26.2$, $23.7,23.6,22.3,22.2,20.8,20.8,16.5,16.5$; IR (neat) $v$ : $814,1080,1410,1580,2960 \mathrm{~cm}^{-1}$. HRMS (ESI) calcd for $\mathrm{C}_{24} \mathrm{H}_{41} \mathrm{~N}_{2} \mathrm{O}_{2}[\mathrm{M}+\mathrm{H}]^{+}: 389.3168$, found 389.3159.

4-(4-Phenylbutoxy)pyridine (4I): Following the general procedure I to afford $4 \mathbf{1}(208.8 \mathrm{mg}, 92 \%$ yield) as colourless oil (eluent: $V($ hexane $/ V($ EtOAc $)=10 / 1) .{ }^{1} \mathrm{H}$ NMR $(400$ $\left.\mathrm{MHz}, \mathrm{CDCl}_{3}\right) \delta: 8.35(\mathrm{~d}, J=5.0 \mathrm{~Hz}, 2 \mathrm{H}), 7.24(\mathrm{t}, J=7.3$ $\mathrm{Hz}, 2 \mathrm{H}), 7.15$ (d, $J=7.0 \mathrm{~Hz}, 3 \mathrm{H}), 6.72$ (d, $J=5.1 \mathrm{~Hz}, 2 \mathrm{H})$, $3.93(\mathrm{~d}, J=5.6 \mathrm{~Hz}, 2 \mathrm{H}), 2.64(\mathrm{t}, J=6.1 \mathrm{~Hz}, 2 \mathrm{H}), 1.77(\mathrm{~s}$, $4 \mathrm{H}) ;{ }^{13} \mathrm{C}$ NMR $\left(101 \mathrm{MHz}, \mathrm{CDCl}_{3}\right) \delta: 165.0,151.0,141.9$, $128.4,128.4,125.9,110.3,67.6,35.5,28.4,27.7$; IR (neat) $v: 818,991,1290,1590,2930 \mathrm{~cm}^{-1}$. HRMS (ESI) calcd for $\mathrm{C}_{15} \mathrm{H}_{18} \mathrm{NO}\left[\mathrm{M}+\mathrm{H}^{+}\right]$: 228.1388, found 228.1385.

(S)-4-(2-Methylbutoxy)pyridine (4m): Following the general procedure I to afford $4 \mathrm{~m}(56.1 \mathrm{mg}, 68 \%$ yield) as colourless oil (eluent: $V($ hexane $/ V($ EtOAc $)=10 / 1) .{ }^{1} \mathrm{H}$ NMR (400 MHz, $\left.\mathrm{CDCl}_{3}\right) \delta: 8.35(\mathrm{~d}, J=5.2 \mathrm{~Hz}, 2 \mathrm{H}), 6.75$ $(\mathrm{d}, J=5.3 \mathrm{~Hz}, 2 \mathrm{H}), 3.85 \sim 3.77(\mathrm{~m}, 1 \mathrm{H}), 3.73(\mathrm{t}, J=7.7$ $\mathrm{Hz}, 1 \mathrm{H}), 1.83$ (q, $J=12.9,6.5 \mathrm{~Hz}, 1 \mathrm{H}), 1.51$ (q, $J=13.4$, $6.7 \mathrm{~Hz}, 1 \mathrm{H}), 1.23$ (q, $J=13.7,7.2 \mathrm{~Hz}, 2 \mathrm{H}), 0.97$ (d, $J=6.7$ $\mathrm{Hz}, 3 \mathrm{H}), 0.90(\mathrm{t}, J=7.4 \mathrm{~Hz}, 3 \mathrm{H}) ;{ }^{13} \mathrm{C}$ NMR $(101 \mathrm{MHz}$, $\left.\mathrm{CDCl}_{3}\right) \delta: 165.4,150.8,110.4,72.7,34.5,26.0,16.4,11.3$; IR (neat) $v: 816,1010,1290,1590,2960,3290 \mathrm{~cm}^{-1}$. HRMS (ESI) calcd for $\mathrm{C}_{10} \mathrm{H}_{16} \mathrm{NO}[\mathrm{M}+\mathrm{H}]^{+}:$166.1232, found 166.1226 .

4-(Heptan-2-yloxy)pyridine (4n): Following the general procedure I to afford $4 \mathrm{n}(84.0 \mathrm{mg}, 87 \%$ yield $)$ as colourless oil (eluent: $V($ hexane $/ V($ EtOAc $)=10 / 1) .{ }^{1} \mathrm{H}$ NMR $(400$ $\left.\mathrm{MHz}, \mathrm{CDCl}_{3}\right) \delta: 8.43 \sim 8.32(\mathrm{~m}, 2 \mathrm{H}), 6.73(\mathrm{~d}, J=5.2 \mathrm{~Hz}$, $2 \mathrm{H}), 4.41(\mathrm{q}, J=6.0 \mathrm{~Hz}, 1 \mathrm{H}), 1.70(\mathrm{q}, J=15.2,7.1 \mathrm{~Hz}$, $1 \mathrm{H}), 1.64 \sim 1.48(\mathrm{~m}, 1 \mathrm{H}), 1.43 \sim 1.24(\mathrm{~m}, 9 \mathrm{H}), 0.86(\mathrm{t}, J=$ $5.8 \mathrm{~Hz}, 3 \mathrm{H}) ;{ }^{13} \mathrm{C} \mathrm{NMR}\left(101 \mathrm{MHz}, \mathrm{CDCl}_{3}\right) \delta: 164.4,151.1$, $111.1,73.9,36.2,31.8,25.1,22.6,19.5,14.1$; IR (neat) $v$ : $818,991,1290,1500,1590,2930,3380 \mathrm{~cm}^{-1}$. HRMS (ESI) calcd for $\mathrm{C}_{12} \mathrm{H}_{20} \mathrm{NO}[\mathrm{M}+\mathrm{H}]^{+}:$194.1545, found 194.1539.

4-(((1R,2S,4S)-1,3,3-Trimethylbicyclo[2.2.1]heptan-2yl)oxy)pyridine (4o): Following the general procedure $\mathbf{I}$ to afford 40 (95.9 mg, 83\% yield) as colourless oil (eluent: $V($ hexane $/ V($ EtOAc $)=10 / 1) .{ }^{1} \mathrm{H}$ NMR $\left(400 \mathrm{MHz}, \mathrm{CDCl}_{3}\right)$ $\delta: 8.34(\mathrm{~d}, J=5.1 \mathrm{~Hz}, 2 \mathrm{H}), 6.77(\mathrm{~d}, J=5.3 \mathrm{~Hz}, 2 \mathrm{H}), 3.92$ 
$(\mathrm{s}, 1 \mathrm{H}), 2.06 \sim 1.88(\mathrm{~m}, 1 \mathrm{H}), 1.70(\mathrm{~d}, J=10.5 \mathrm{~Hz}, 2 \mathrm{H})$, $1.58(\mathrm{~d}, J=10.4 \mathrm{~Hz}, 1 \mathrm{H}), 1.44$ (q, $J=12.4,6.1 \mathrm{~Hz}, 1 \mathrm{H})$, $1.19(\mathrm{~d}, J=21.7 \mathrm{~Hz}, 4 \mathrm{H}), 1.05(\mathrm{~s}, 4 \mathrm{H}), 0.76(\mathrm{~s}, 3 \mathrm{H}) ;{ }^{13} \mathrm{C}$ NMR (101 MHz, $\left.\mathrm{CDCl}_{3}\right) \delta$ : 166.0, 151.0, 111.1, 89.6, $49.5,49.1,41.4,40.2,30.5,26.4,25.8,20.2$, 19.8; IR (neat) $v: 816,1050,1290,1500,1590,2960 \mathrm{~cm}^{-1}$. HRMS (ESI) calcd for $\mathrm{C}_{15} \mathrm{H}_{22} \mathrm{NO}[\mathrm{M}+\mathrm{H}]^{+}: 232.1701$, found 232.1689 .

(1S,5S)-8-Methyl-3-(pyridin-2-yloxy)-8-azabicyclo[3.2. 1]octane (4p): Following the general procedure $I$ to afford 4p (94.8 mg, 87\% yield) as colourless oil (eluent: $V$ (hexa$\mathrm{ne} / V($ EtOAc $)=2 / 1) .{ }^{1} \mathrm{H}$ NMR $\left(400 \mathrm{MHz}, \mathrm{CDCl}_{3}\right) \delta: 8.03$ $(\mathrm{d}, J=3.9 \mathrm{~Hz}, 1 \mathrm{H}), 7.43(\mathrm{t}, J=7.6 \mathrm{~Hz}, 1 \mathrm{H}), 6.72(\mathrm{t},-+J=$ $5.9 \mathrm{~Hz}, 1 \mathrm{H}), 6.58(\mathrm{~d}, J=8.3 \mathrm{~Hz}, 1 \mathrm{H}), 5.24$ (q, $J=11.2,6.2$ $\mathrm{Hz}, 1 \mathrm{H}), 3.16(\mathrm{~s}, 2 \mathrm{H}), 2.29(\mathrm{~s}, 3 \mathrm{H}), 1.97(\mathrm{t}, J=11.1 \mathrm{~Hz}$, $4 \mathrm{H}), 1.79 \sim 1.64(\mathrm{~m}, 4 \mathrm{H}) ;{ }^{13} \mathrm{C}$ NMR $\left(101 \mathrm{MHz}, \mathrm{CDCl}_{3}\right) \delta$ : $163.4,146.8,138.4,116.3,111.6,67.4,60.3,38.4,35.6$, 26.8; IR (neat) $v: 793,1050,1290,1430,1590,2930 \mathrm{~cm}^{-1}$. HRMS (ESI) calcd for $\mathrm{C}_{13} \mathrm{H}_{19} \mathrm{~N}_{2} \mathrm{O}[\mathrm{M}+\mathrm{H}]^{+}: 219.1497$, found 219.1486 .

$(1 S, 3 R, 4 S)$-3-(Pyridin-4-yloxy)quinuclidine (4q): Following the general procedure I to afford $\mathbf{4 q}(89.8 \mathrm{mg}, 88 \%$ yield) as colourless oil (eluent: $V($ hexane $/ V($ EtOAc $)=$ 10/1). ${ }^{1} \mathrm{H}$ NMR (400 MHz, $\left.\mathrm{CDCl}_{3}\right) \delta: 8.33(\mathrm{~d}, J=5.5 \mathrm{~Hz}$, 2H), 6.68 (d, $J=5.5 \mathrm{~Hz}, 2 \mathrm{H}), 4.37$ (d, $J=7.3 \mathrm{~Hz}, 1 \mathrm{H}), 3.22$ (dd, $J=14.4,8.0 \mathrm{~Hz}, 1 \mathrm{H}), 2.92 \sim 2.66(\mathrm{~m}, 5 \mathrm{H}), 2.09$ (s, $1 \mathrm{H}), 1.88(\mathrm{t}, J=11.4 \mathrm{~Hz}, 1 \mathrm{H}), 1.78 \sim 1.61(\mathrm{~m}, 1 \mathrm{H}), 1.51(\mathrm{~s}$, $1 \mathrm{H}), 1.41 \sim 1.29(\mathrm{~m}, 1 \mathrm{H}),{ }^{13} \mathrm{C}$ NMR $\left(101 \mathrm{MHz}, \mathrm{CDCl}_{3}\right) \delta$ : 163.7, 151.1, 111.0, 73.7, 55.4, 47.2, 46.5, 25.0, 24.3, 19.1; IR (neat) $v: 1020,1280,1590,2850,2920 \mathrm{~cm}^{-1}$. HRMS (ESI) calcd for $\mathrm{C}_{12} \mathrm{H}_{17} \mathrm{~N}_{2} \mathrm{O}[\mathrm{M}+\mathrm{H}]^{+}:$205.1341, found 205.1334 .

4-(((1S,2S,4S)-1,7,7-Trimethylbicyclo[2.2.1]heptan-2yl)oxy)pyridine (4r): Following the general procedure I to afford 4r (108.6 mg, 94\% yield) as white solid (eluent: $V($ hexane $/ V($ EtOAc $)=10 / 1)$. m.p. $67.9{ }^{\circ} \mathrm{C} ;{ }^{1} \mathrm{H}$ NMR $(400$ $\left.\mathrm{MHz}, \mathrm{CDCl}_{3}\right) \delta: 8.37(\mathrm{~d}, J=5.1 \mathrm{~Hz}, 2 \mathrm{H}), 6.72(\mathrm{~d}, J=5.3$ $\mathrm{Hz}, 2 \mathrm{H}), 4.37$ (d, $J=8.9 \mathrm{~Hz}, 1 \mathrm{H}), 2.39$ (q, $J=9.6,4.7 \mathrm{~Hz}$, $1 \mathrm{H}), 2.22 \sim 2.13(\mathrm{~m}, 1 \mathrm{H}), 1.77(\mathrm{t}, J=7.0 \mathrm{~Hz}, 2 \mathrm{H}), 1.35(\mathrm{t}$, $J=12.0 \mathrm{~Hz}, 1 \mathrm{H}), 1.24(\mathrm{t}, J=10.1 \mathrm{~Hz}, 1 \mathrm{H}), 1.07(\mathrm{~d}, J=$ $13.4 \mathrm{~Hz}, 1 \mathrm{H}), 0.94(\mathrm{~s}, 9 \mathrm{H}) ;{ }^{13} \mathrm{C} \mathrm{NMR}\left(101 \mathrm{MHz}, \mathrm{CDCl}_{3}\right) \delta$ : 165.0, 150.9, 111.2, 83.1, 49.5, 47.7, 45.1, 36.5, 27.9, 26.7, $19.7,18.9,13.7$; IR (neat) $v: 816,1020,1280,1500,1590$, $2950 \mathrm{~cm}^{-1}$. HRMS (ESI) calcd for $\mathrm{C}_{15} \mathrm{H}_{22} \mathrm{NO}[\mathrm{M}+\mathrm{H}]^{+}$: 232.1701 , found 232.1688 .

4-(Heptan-2-yloxy)-2-methylquinoline (4s): Following the general procedure I to afford $4 \mathbf{s}$ (93.8 $\mathrm{mg}, 73 \%$ yield) as colourless oil (eluent: $V($ hexane $/ V($ EtOAc $)=4 / 1) .{ }^{1} \mathrm{H}$ NMR (400 MHz, $\left.\mathrm{CDCl}_{3}\right) \delta: 8.14(\mathrm{~d}, J=8.3 \mathrm{~Hz}, 1 \mathrm{H}), 7.93$ $(\mathrm{d}, J=8.5 \mathrm{~Hz}, 1 \mathrm{H}), 7.63(\mathrm{t}, J=7.6 \mathrm{~Hz}, 1 \mathrm{H}), 7.40(\mathrm{t}, J=7.6$ $\mathrm{Hz}, 1 \mathrm{H}), 6.58(\mathrm{~s}, 1 \mathrm{H}), 4.63$ (q, $J=6.0 \mathrm{~Hz}, 1 \mathrm{H}), 2.68$ (s, $3 \mathrm{H}), 1.86$ (q, $J=12.5,7.3,5.9 \mathrm{~Hz}, 1 \mathrm{H}), 1.70$ (q, $J=14.3$, $10.8,5.5 \mathrm{~Hz}, 1 \mathrm{H}), 1.53 \sim 1.39(\mathrm{~m}, 5 \mathrm{H}), 1.35 \sim 1.29(\mathrm{~m}$, $4 \mathrm{H}), 0.88$ (t, $J=6.6 \mathrm{~Hz}, 3 \mathrm{H}) ;{ }^{13} \mathrm{C} \mathrm{NMR}\left(101 \mathrm{MHz}, \mathrm{CDCl}_{3}\right)$ $\delta: 161.1,160.0,149.0,129.8,128.0,124.6,122.0,120.6$, $101.7,74.4,36.3,31.8,26.0,25.2,22.7,19.5,14.1$; IR (neat) $v: 764,1110,1420,1590,2930 \mathrm{~cm}^{-1}$. HRMS (ESI) calcd for $\mathrm{C}_{17} \mathrm{H}_{24} \mathrm{NO}[\mathrm{M}+\mathrm{H}]^{+}: 258.1858$, found 258.1853 .

4-(tert-Butoxy)-2-(trifluoromethyl) quinoline (4t): Following the general procedure I to afford $4 t(50.8 \mathrm{mg}, 39 \%$ yield) as yellow oil (eluent: $V($ hexane $/ V($ EtOAc $)=50 / 1)$. ${ }^{1} \mathrm{H}$ NMR (400 MHz, $\left.\mathrm{CDCl}_{3}\right) \delta: 8.22(\mathrm{~d}, J=8.4 \mathrm{~Hz}, 1 \mathrm{H})$, $8.12(\mathrm{~d}, J=8.5 \mathrm{~Hz}, 1 \mathrm{H}), 7.74(\mathrm{t}, J=7.6 \mathrm{~Hz}, 1 \mathrm{H}), 7.57$ (t, $J=7.6 \mathrm{~Hz}, 1 \mathrm{H}), 7.21(\mathrm{~s}, 1 \mathrm{H}), 1.67(\mathrm{~s}, 9 \mathrm{H}) ;{ }^{13} \mathrm{C}$ NMR $(101$ $\left.\mathrm{MHz}, \mathrm{CDCl}_{3}\right) \delta: 161.2,148.7,148.6(\mathrm{q}, J=34.0 \mathrm{~Hz})$, $130.8,129.8,127.3,124.3,122.8,122.3$ (q, $J=274.0 \mathrm{~Hz})$, 101.64, 81.93, 28.71; ${ }^{19} \mathrm{~F}$ NMR $\left(376 \mathrm{MHz} \mathrm{CDCl}_{3}\right) \delta$ : -67.77; IR (neat) $v: 768,941,1130,1390,2920 \mathrm{~cm}^{-1}$. HRMS (ESI) calcd for $\mathrm{C}_{14} \mathrm{H}_{14} \mathrm{~F}_{3} \mathrm{NONa}[\mathrm{M}+\mathrm{Na}]^{+}$: 292.0925, found 292.0923.

2-(( $\left(4 S, 5^{\prime} R, 6 \mathrm{a} R, 6 \mathrm{~b} S, 8 \mathrm{a} S, 8 \mathrm{~b} R, 9 S, 10 R, 11 \mathrm{a} S, 12 \mathrm{a} S, 12 \mathrm{~b} S\right)-$ 5',6a,8a,9-Tetramethyl-1,3,3',4,4',5,5',6,6a,6b,6',7,8,8a,8b, 9,11a,12,12a,12b-icosahydrospiro[naphtho[2', $1^{\prime}: 4,5$ ]indeno[2,1-b]furan-10,2'-pyran]-4-yl)oxy)pyridine (4u): Following the general procedure II to afford $\mathbf{4 u}(85.4 \mathrm{mg}$, $87 \%$ yield) as white solid (eluent: $V($ hexane $/ V($ EtOAc $)=$ 100/1). m.p. $227{ }^{\circ} \mathrm{C} ;{ }^{1} \mathrm{H}$ NMR (400 MHz, $\mathrm{CDCl}_{3}$ ) $\delta: 8.12$ $(\mathrm{d}, J=4.5 \mathrm{~Hz}, 1 \mathrm{H}), 7.53(\mathrm{t}, J=7.5 \mathrm{~Hz}, 1 \mathrm{H}), 6.81(\mathrm{t}, J=5.6$ $\mathrm{Hz}, 1 \mathrm{H}), 6.67$ (d, $J=8.2 \mathrm{~Hz}, 1 \mathrm{H}), 5.41(\mathrm{~s}, 1 \mathrm{H}), 4.90(\mathrm{t}, J=$ $11.3 \mathrm{~Hz}, 1 \mathrm{H}), 4.42$ (q, $J=7.2 \mathrm{~Hz}, 1 \mathrm{H}), 3.47$ (d, $J=8.6 \mathrm{~Hz}$, $1 \mathrm{H}), 3.38$ (t, $J=10.8 \mathrm{~Hz}, 1 \mathrm{H}), 2.53$ (d, $J=12.7 \mathrm{~Hz}, 1 \mathrm{H})$, $2.39(\mathrm{t}, J=12.2 \mathrm{~Hz}, 1 \mathrm{H}), 2.10 \sim 1.94(\mathrm{~m}, 3 \mathrm{H}), 1.93 \sim 1.81$ $(\mathrm{m}, 2 \mathrm{H}), 1.67$ (q, $J=39.4,31.0,26.4,12.4 \mathrm{~Hz}, 14 \mathrm{H})$, $1.32 \sim 1.06(\mathrm{~m}, 7 \mathrm{H}), 0.98(\mathrm{~d}, J=6.5 \mathrm{~Hz}, 3 \mathrm{H}), 0.79(\mathrm{~d}, J=$ $5.4 \mathrm{~Hz}, 6 \mathrm{H}) ;{ }^{13} \mathrm{C}$ NMR $\left(101 \mathrm{MHz}, \mathrm{CDCl}_{3}\right) \delta: 163.5,147.0$, $140.5,138.6,122.0,116.4,111.6,109.4,81.0,74.4,66.94$, $62.3,56.6,50.2,41.7,40.4,39.9,38.6,37.3,37.0,32.2$, $32.0,31.6,31.5,30.4,28.9,28.2,21.0,19.6,17.3,16.4$, 14.7; IR (neat) $v: 810,964,1122,1320,1450,1580,2850$, $2920 \mathrm{~cm}^{-1}$. HRMS (ESI) calcd for $\mathrm{C}_{32} \mathrm{H}_{46} \mathrm{NO}_{3}[\mathrm{M}+\mathrm{H}]^{+}$: 492.3478, found 492.3477 .

2-(((3S,8S,9S,10R,13R,14S,17R)-10,13-dimethyl-17$((R)-6$-Methylheptan-2-yl)-2,3,4,7,8,9,10,11,12,13,14,15, 16,17-tetradecahydro-1H-cyclopenta[a]phenanthren-3-yl)oxy)pyridine (4v): Following the general procedure II to afford $4 \mathbf{v}$ (80.6 mg, $87 \%$ yield) as white solid (eluent: $V($ hexane $/ V($ EtOAc $)=100 / 1)$. m.p. $161{ }^{\circ} \mathrm{C} ;{ }^{1} \mathrm{H}$ NMR $(400$ $\left.\mathrm{MHz}, \mathrm{CDCl}_{3}\right) \delta: 8.12(\mathrm{~d}, J=5.0 \mathrm{~Hz}, 1 \mathrm{H}), 7.51(\mathrm{t}, J=7.7$ $\mathrm{Hz}, 1 \mathrm{H}), 6.83 \sim 6.72(\mathrm{~m}, 1 \mathrm{H}), 6.67(\mathrm{~d}, J=8.3 \mathrm{~Hz}, 1 \mathrm{H})$, $5.40(\mathrm{~s}, 1 \mathrm{H}), 4.90(\mathrm{dd}, J=10.7,5.6 \mathrm{~Hz}, 1 \mathrm{H}), 2.52(\mathrm{~d}, J=$ $12.8 \mathrm{~Hz}, 1 \mathrm{H}), 2.40(\mathrm{t}, J=12.0 \mathrm{~Hz}, 1 \mathrm{H}), 2.00(\mathrm{t}, J=15.0$ $\mathrm{Hz}, 3 \mathrm{H}), 1.92 \sim 1.79(\mathrm{~m}, 2 \mathrm{H}), 1.75 \sim 1.44(\mathrm{~m}, 7 \mathrm{H}), 1.39 \sim$ $0.99(\mathrm{~m}, 17 \mathrm{H}), 0.93(\mathrm{~d}, J=6.3 \mathrm{~Hz}, 3 \mathrm{H}), 0.87(\mathrm{~d}, J=6.6$ $\mathrm{Hz}, 6 \mathrm{H}), 0.69(\mathrm{~s}, 3 \mathrm{H}) ;{ }^{13} \mathrm{C} \mathrm{NMR}\left(101 \mathrm{MHz}, \mathrm{CDCl}_{3}\right) \delta$ : $163.5,147.1,140.4,138.5,122.3,116.4,111.5,74.5,56.9$, $56.3,50.3,42.5,39.9,39.7,38.7,37.3,36.9,36.4,35.9$, $32.1,32.1,28.4,28.2,28.1,24.4,24.0,23.0,22.7,21.2$, 19.6, 18.9, 12.0; IR (neat) $v: 1290,1430,1470,1570,2940$ $\mathrm{cm}^{-1}$. HRMS (ESI) calcd for $\mathrm{C}_{32} \mathrm{H}_{51} \mathrm{NO}[\mathrm{M}+\mathrm{H}]^{+}$: 464.3892, found 464.3886 .

2-(((3S,9S,10R,13R,14R,17R)-17-((2R,5R,E)-5,6-Dimethylhept-3-en-2-yl)-10,13-dimethyl-2,3,4,9,10,11,12,13,14, 15,16,17-dodecahydro-1H-cyclopenta[a]phenanthren-3yl)oxy)pyridine (4w): Following the general procedure II 
to afford $4 \mathbf{w}(65.3 \mathrm{mg}, 69 \%$ yield) as white solid (eluent: $V($ hexane $/ V($ EtOAc $)=100 / 1)$. m.p. $160{ }^{\circ} \mathrm{C} ;{ }^{1} \mathrm{H}$ NMR $(400$ $\left.\mathrm{MHz}, \mathrm{CDCl}_{3}\right) \delta: 8.19 \sim 8.09(\mathrm{~m}, 1 \mathrm{H}), 7.54(\mathrm{t}, J=8.3 \mathrm{~Hz}$, $1 \mathrm{H}), 6.86 \sim 6.78(\mathrm{~m}, 1 \mathrm{H}), 6.68(\mathrm{~d}, J=8.3 \mathrm{~Hz}, 1 \mathrm{H}), 5.64 \sim$ $5.56(\mathrm{~m}, 1 \mathrm{H}), 5.45 \sim 5.36(\mathrm{~m}, 1 \mathrm{H}), 5.27 \sim 5.13(\mathrm{~m}, 2 \mathrm{H})$, $5.00(\mathrm{q}, J=11.2,5.5 \mathrm{~Hz}, 1 \mathrm{H}), 2.70(\mathrm{dd}, J=13.3,3.2 \mathrm{~Hz}$, $1 \mathrm{H}), 2.46(\mathrm{t}, J=12.8 \mathrm{~Hz}, 1 \mathrm{H}), 2.11 \sim 2.02(\mathrm{~m}, 3 \mathrm{H}), 1.95 \sim$ $1.83(\mathrm{~m}, 3 \mathrm{H}), 1.70(\mathrm{q}, J=37.5,15.9,7.9 \mathrm{~Hz}, 5 \mathrm{H}), 1.50 \sim$ $1.24(\mathrm{~m}, 7 \mathrm{H}), 1.05$ (d, $J=6.6 \mathrm{~Hz}, 3 \mathrm{H}), 1.00(\mathrm{~s}, 3 \mathrm{H}), 0.93$ (d, $J=6.8 \mathrm{~Hz}, 3 \mathrm{H}), 0.84$ (t, $J=6.3 \mathrm{~Hz}, 6 \mathrm{H}), 0.64$ (s, 3H); ${ }^{13} \mathrm{C}$ NMR $\left(101 \mathrm{MHz}, \mathrm{CDCl}_{3}\right) \delta: 163.5,147.1,141.4,139.6$, 138.6, 135.7, 132.1, 120.1, 116.6, 116.5, 111.6, 73.3, 55.9, $54.7,46.3,43.0,40.6,39.3,38.3,37.4,37.3,33.2,28.5$, $28.5,23.2,21.3,21.2,20.1,19.8,17.8,16.4,12.2$; IR (neat) $v: 783,1290,1360,1430,1470,2930 \mathrm{~cm}^{-1}$. HRMS (ESI) calcd for $\mathrm{C}_{33} \mathrm{H}_{47} \mathrm{NONa}[\mathrm{M}+\mathrm{Na}]^{+}: 496.3555$, found 496.3538.

Methyl (4aS,6aS,6b $R, 8 \mathrm{a} R, 10 S, 12 \mathrm{a} R, 12 \mathrm{~b} R, 14 \mathrm{~b} S)-2,2,6 \mathrm{a}$, $6 \mathrm{~b}, 9,9,12 \mathrm{a}-$ heptamethyl-10-(pyridin-2-yloxy)-1,3,4,5,6,6a, $6 \mathrm{~b}, 7,8,8 \mathrm{a}, 9,10,11,12,12 \mathrm{a}, 12 \mathrm{~b}, 13,14 \mathrm{~b}$-octadecahydropicene$\mathbf{4 a}(2 H)$-carboxylate $(\mathbf{4 x})$ : Following the general procedure II to afford $4 \mathbf{x}$ (56.9 mg, 52\% yield) as white solid (eluent: $V($ hexane $/ V($ EtOAc $)=100 / 1)$. m.p. $187.8{ }^{\circ} \mathrm{C} ;{ }^{1} \mathrm{H}$ NMR $\left(400 \mathrm{MHz}, \mathrm{CDCl}_{3}\right) \delta: 8.09(\mathrm{~d}, J=4.7 \mathrm{~Hz}, 1 \mathrm{H}), 7.50(\mathrm{t}, J=$ $7.7 \mathrm{~Hz}, 1 \mathrm{H}), 6.84 \sim 6.71(\mathrm{~m}, 1 \mathrm{H}), 6.71 \sim 6.57(\mathrm{~m}, 1 \mathrm{H}), 5.28$ (s, 1H), $4.78(\mathrm{dd}, J=11.5,4.2 \mathrm{~Hz}, 1 \mathrm{H}), 3.61(\mathrm{~s}, 3 \mathrm{H}), 2.86$ (d, $J=13.7 \mathrm{~Hz}, 1 \mathrm{H}), 1.89$ (q, $J=27.7,6.6 \mathrm{~Hz}, 4 \mathrm{H}), 1.72 \sim$ $1.40(\mathrm{~m}, 11 \mathrm{H}), 1.38 \sim 1.24(\mathrm{~m}, 4 \mathrm{H}), 1.13(\mathrm{~d}, J=9.8 \mathrm{~Hz}$, $5 \mathrm{H}), 0.97 \sim 0.87(\mathrm{~m}, 16 \mathrm{H}), 0.73(\mathrm{~s}, 3 \mathrm{H}) ;{ }^{13} \mathrm{C}$ NMR $(101$ $\left.\mathrm{MHz}, \mathrm{CDCl}_{3}\right) \delta: 178.3,164.4,146.9,143.9,138.5,122.5$, 116.1, 111.7, 81.3, 55.7, 51.6, 47.7, 46.8, 45.9, 41.7, 41.4, $39.4,38.4,38.3,37.2,34.0,33.2,32.8,32.5,30.8,28.3$, $27.8,26.0,23.8,23.6,23.3,23.2,18.4,17.0,17.0,15.5$; IR (neat) $v: 883,989,1290,1470,1600,1730,2940 \mathrm{~cm}^{-1}$. HRMS (ESI) calcd for $\mathrm{C}_{36} \mathrm{H}_{54} \mathrm{NO}_{3}[\mathrm{M}+\mathrm{H}]^{+}:$548.4104, found 548.4088 .

2-(((8R,9S,13S,14S,17S)-3-Methoxy-13-methyl-7,8,9, $11,12,13,14,15,16,17$-decahydro- $6 H$-cyclopenta[a]phenanthren-17-yl)oxy)pyridine (4y): Following the general procedure II to afford $\mathbf{4 y}(71.2 \mathrm{mg}, 98 \%$ yield) as white solid (eluent: $V($ hexane $/ V($ EtOAc $)=100 / 1)$. m.p. $139.6{ }^{\circ} \mathrm{C} ;{ }^{1} \mathrm{H}$ NMR (400 MHz, $\left.\mathrm{CDCl}_{3}\right) \delta: 8.16(\mathrm{~d}, J=4.2 \mathrm{~Hz}, 1 \mathrm{H}), 7.55$ $(\mathrm{t}, J=7.6 \mathrm{~Hz}, 1 \mathrm{H}), 7.22(\mathrm{~d}, J=8.6 \mathrm{~Hz}, 1 \mathrm{H}), 6.85(\mathrm{~s}, 1 \mathrm{H})$, $6.74(\mathrm{~d}, J=7.9 \mathrm{~Hz}, 2 \mathrm{H}), 6.66(\mathrm{~s}, 1 \mathrm{H}), 4.99(\mathrm{t}, J=8.3 \mathrm{~Hz}$, $1 \mathrm{H}), 3.79(\mathrm{~s}, 3 \mathrm{H}), 2.95 \sim 2.80(\mathrm{~m}, 2 \mathrm{H}), 2.48 \sim 2.35(\mathrm{~m}$, $1 \mathrm{H}), 2.26(\mathrm{q}, J=9.8 \mathrm{~Hz}, 2 \mathrm{H}), 1.95(\mathrm{~d}, J=11.1 \mathrm{~Hz}, 2 \mathrm{H})$, 1.81 (q, $J=7.7,7.1 \mathrm{~Hz}, 1 \mathrm{H}), 1.67 \sim 1.36(\mathrm{~m}, 7 \mathrm{H}), 0.97(\mathrm{~s}$, $3 \mathrm{H}) ;{ }^{13} \mathrm{C} \mathrm{NMR}\left(101 \mathrm{MHz}, \mathrm{CDCl}_{3}\right) \delta: 164.4,157.5,147.0$, $138.4,138.1,132.8,126.4,116.4,113.9,111.6,111.5$, 83.7, 55.3, 49.9, 44.1, 43.3, 38.8, 37.4, 30.0, 28.1, 27.4, 26.5, 23.5, 12.3; IR (neat) $v: 777,1010,1290,1430,1600$, $2920 \mathrm{~cm}^{-1}$. HRMS (ESI) calcd for $\mathrm{C}_{24} \mathrm{H}_{30} \mathrm{NO}_{2}[\mathrm{M}+\mathrm{H}]^{+}$: 364.2277, found 364.2269.

2-(((3S,8R,9S,10R,13S,14S)-10,13-Dimethyl-17-(pyridin-3-yl)-2,3,4,7,8,9,10,11,12,13,14,15-dodecahydro- $1 H$ cyclopenta[a]phenanthren-3-yl)oxy)pyridine (4z): Following the general procedure II to afford $\mathbf{4 z}(69.0 \mathrm{mg}, 81 \%$ yield) as white solid (eluent: $V($ hexane $/ V($ EtOAc $)=1 / 1)$. m.p. $145{ }^{\circ} \mathrm{C} ;{ }^{1} \mathrm{H}$ NMR $\left(400 \mathrm{MHz}, \mathrm{CDCl}_{3}\right) \delta$ : $8.62(\mathrm{~s}, 1 \mathrm{H})$, $8.46(\mathrm{~s}, 1 \mathrm{H}), 8.12(\mathrm{~d}, J=4.6 \mathrm{~Hz}, 1 \mathrm{H}), 7.65(\mathrm{~d}, J=7.8 \mathrm{~Hz}$, $1 \mathrm{H}), 7.53(\mathrm{t}, J=7.5 \mathrm{~Hz}, 1 \mathrm{H}), 7.25 \sim 7.17(\mathrm{~m}, 1 \mathrm{H}), 6.81(\mathrm{t}$, $J=5.9 \mathrm{~Hz}, 1 \mathrm{H}), 6.67(\mathrm{~d}, J=8.3 \mathrm{~Hz}, 1 \mathrm{H}), 5.99$ (s, 1H), 5.45 $(\mathrm{s}, 1 \mathrm{H}), 4.92(\mathrm{q}, J=11.2,6.1 \mathrm{~Hz}, 1 \mathrm{H}), 2.56(\mathrm{~d}, J=14.5 \mathrm{~Hz}$, $1 \mathrm{H}), 2.41(\mathrm{t}, J=12.2 \mathrm{~Hz}, 1 \mathrm{H}), 2.26(\mathrm{t}, J=7.6 \mathrm{~Hz}, 1 \mathrm{H})$, $2.10 \sim 2.01(\mathrm{~m}, 4 \mathrm{H}), 1.90(\mathrm{~d}, J=13.1 \mathrm{~Hz}, 1 \mathrm{H}), 1.68(\mathrm{q}, J=$ $36.1,23.1,11.4 \mathrm{~Hz}, 6 \mathrm{H}), 1.54 \sim 1.38(\mathrm{~m}, 2 \mathrm{H}), 1.08(\mathrm{~d}, J=$ $28.5 \mathrm{~Hz}, 7 \mathrm{H}) ;{ }^{13} \mathrm{C} \mathrm{NMR}\left(101 \mathrm{MHz}, \mathrm{CDCl}_{3}\right) \delta$ : 163.4, $151.8,148.0,147.9,147.0,140.8,138.5,133.7,133.0$, $129.3,123.1,121.9,116.4,111.5,74.3,57.6,50.5,47.4$, $38.6,37.2,37.0,35.3,31.9,31.6,30.5,28.1,20.9,19.4$, 16.7; IR (neat) $v: 885,989,1290,1430,1470,2920 \mathrm{~cm}^{-1}$. HRMS (ESI) calcd for $\mathrm{C}_{29} \mathrm{H}_{35} \mathrm{~N}_{2} \mathrm{O}[\mathrm{M}+\mathrm{H}]^{+}:$427.2749, found 427.2736 .

2-Chloro-10-(3-(4-(2-(pyridin-2-yloxy)ethyl)piperazin1-yl)propyl)-10H-phenothiazine (4aa): Following the general procedure II to afford 4aa (79.7 $\mathrm{mg}, 83 \%$ yield) as white solid (eluent: $V($ hexane $/ V($ EtOAc $)=1 / 1)$. m.p. $160{ }^{\circ} \mathrm{C} ;{ }^{1} \mathrm{H}$ NMR $\left(400 \mathrm{MHz}, \mathrm{CDCl}_{3}\right) \delta: 8.12$ (d, $J=4.7$ $\mathrm{Hz}, 1 \mathrm{H}), 7.53$ (t, $J=7.7 \mathrm{~Hz}, 1 \mathrm{H}), 7.11(\mathrm{dd}, J=17.3,8.0$ $\mathrm{Hz}, 2 \mathrm{H}), 6.98(\mathrm{~d}, J=8.0 \mathrm{~Hz}, 1 \mathrm{H}), 6.94 \sim 6.79(\mathrm{~m}, 5 \mathrm{H})$, $6.73(\mathrm{~d}, J=8.4 \mathrm{~Hz}, 1 \mathrm{H}), 4.42(\mathrm{t}, J=5.8 \mathrm{~Hz}, 2 \mathrm{H}), 3.86(\mathrm{t}$, $J=6.8 \mathrm{~Hz}, 2 \mathrm{H}), 2.77(\mathrm{t}, J=5.8 \mathrm{~Hz}, 2 \mathrm{H}), 2.72 \sim 2.31(\mathrm{~m}$, $10 \mathrm{H}), 1.91(\mathrm{p}, J=6.6 \mathrm{~Hz}, 2 \mathrm{H}) ;{ }^{13} \mathrm{C} \mathrm{NMR}(101 \mathrm{MHz}$, $\left.\mathrm{CDCl}_{3}\right) \delta: 163.7,146.9,146.5,144.6,138.5,133.2,127.9$, $127.5,127.5,124.8,123.5,122.9,122.3,116.8,115.9$, $115.9,111.3,63.3,57.2,55.5,53.5,53.2,45.4,24.3$; IR (neat) $v: 779,1290,1460,1590,2930 \mathrm{~cm}^{-1}$. HRMS (ESI) calcd for $\mathrm{C}_{26} \mathrm{H}_{30} \mathrm{ClN}_{4} \mathrm{OS}[\mathrm{M}+\mathrm{H}]^{+}$: 481.1829, found 481.1827.

4-Phenylcyclohexan-1-d-1-ol (S1): Following the general procedure III to afford S1 (147.9 $\mathrm{mg}, 83 \%$ yield) as white solid (eluent: $V($ hexane $/ V($ EtOAc $)=10 / 1)$. m.p. $122.2{ }^{\circ} \mathrm{C} ;{ }^{1} \mathrm{H}$ NMR $\left(400 \mathrm{MHz}, \mathrm{CDCl}_{3}\right) \delta: 7.34(\mathrm{t}, J=7.5$ $\mathrm{Hz}, 2 \mathrm{H}), 7.24(\mathrm{t}, J=7.7 \mathrm{~Hz}, 3 \mathrm{H}), 2.55(\mathrm{t}, J=11.9 \mathrm{~Hz}, 1 \mathrm{H})$, $2.15(\mathrm{~d}, J=12.1 \mathrm{~Hz}, 2 \mathrm{H}), 1.99$ (d, $J=12.9 \mathrm{~Hz}, 2 \mathrm{H}), 1.73-$ $1.65(\mathrm{~m}, 1 \mathrm{H}), 1.57(\mathrm{t}, J=12.1 \mathrm{~Hz}, 2 \mathrm{H}), 1.48(\mathrm{t}, J=12.7$ $\mathrm{Hz}, 2 \mathrm{H}) ;{ }^{13} \mathrm{C}$ NMR $\left(101 \mathrm{MHz}, \mathrm{CDCl}_{3}\right) \delta: 146.7,128.5$, $126.89,126.2,70.3(\mathrm{t}, J=21.5 \mathrm{~Hz}), 43.5,36.0,32.6$; IR (neat) $v: 3426,3380,3028,2922,2851,1600,1494,1453$, 1124, 1091, 1064, 945, 755, $698 \mathrm{~cm}^{-1}$. HRMS (ESI) calcd for $\mathrm{C}_{12} \mathrm{H}_{15} \mathrm{DONa}[\mathrm{M}+\mathrm{Na}]^{+}: 200.1162$, found 200.1148 .

Cyclooctan-1-d-1-ol (S2): Following the general procedure III to afford S2 (109.1 mg, 85\% yield) as colorless liquid (eluent: $V($ hexane $/ V($ EtOAc $)=25 / 1) .{ }^{1} \mathrm{H}$ NMR $(400$ $\left.\mathrm{MHz}, \mathrm{CDCl}_{3}\right) \delta: 1.86 \sim 1.76(\mathrm{~m}, 2 \mathrm{H}), 1.67(\mathrm{~d}, J=6.6 \mathrm{~Hz}$, $2 \mathrm{H}), 1.63(\mathrm{~d}, J=13.7 \mathrm{~Hz}, 2 \mathrm{H}), 1.58 \sim 1.47(\mathrm{~m}, 7 \mathrm{H}), 1.46 \sim$ $1.41(\mathrm{~m}, 2 \mathrm{H}) ;{ }^{13} \mathrm{C}$ NMR $\left(101 \mathrm{MHz}, \mathrm{CDCl}_{3}\right) \delta: 71.8(\mathrm{t}, J=$ $21.7 \mathrm{~Hz}$ ), 34.7, 27.5, 25.4, 22.8; IR (neat) $v: 3347,2922$, 2851, 1445, $1068 \mathrm{~cm}^{-1}$. HRMS (ESI) calcd for $\mathrm{C}_{8} \mathrm{H}_{15} \mathrm{DO}$ $\mathrm{Na}[\mathrm{M}+\mathrm{Na}]^{+}: 152.1162$, found 152.1158 .

4'-Propyl-[1,1'-bi(cyclohexan)]-4-d-4-ol (S3): Following the general procedure III to afford S3 (173.4 mg, 77\% yield) as white solid (eluent: $V($ hexane $/ V($ EtOAc $)=25 / 1)$. m.p. $125.2{ }^{\circ} \mathrm{C} ;{ }^{1} \mathrm{H} \mathrm{NMR}\left(400 \mathrm{MHz}, \mathrm{CDCl}_{3}\right) \delta: 1.96(\mathrm{~d}, J=$ 
$11.4 \mathrm{~Hz}, 2 \mathrm{H}), 1.70(\mathrm{dd}, J=20.6,12.8 \mathrm{~Hz}, 6 \mathrm{H}), 1.56 \sim 1.45$ $(\mathrm{m}, 1 \mathrm{H}), 1.28(\mathrm{dt}, J=13.5,6.9 \mathrm{~Hz}, 2 \mathrm{H}), 1.19(\mathrm{t}, J=11.3$ $\mathrm{Hz}, 2 \mathrm{H}), 1.12$ (t, $J=5.8 \mathrm{~Hz}, 3 \mathrm{H}), 1.00(\mathrm{~d}, J=10.2 \mathrm{~Hz}, 4 \mathrm{H})$, $0.93(\mathrm{t}, J=12.6 \mathrm{~Hz}, 2 \mathrm{H}), 0.86(\mathrm{t}, J=7.3 \mathrm{~Hz}, 5 \mathrm{H}) ;{ }^{13} \mathrm{C}$ NMR $\left(101 \mathrm{MHz}, \mathrm{CDCl}_{3}\right) \delta: 71.1(\mathrm{t}, J=21.5 \mathrm{~Hz}), 43.0$, $42.5,39.9,37.7,36.0,33.7,30.4,28.3,20.2,14.6$; IR (neat) $v: 3409,2957,2913,2853,1637,1453 \mathrm{~cm}^{-1}$. HRMS (ESI) calcd for $\mathrm{C}_{15} \mathrm{H}_{27} \mathrm{DONa}[\mathrm{M}+\mathrm{Na}]^{+}: 248.2101$, found 248.2097 .

$(1 S, 2 R, 4 S)$-1,7,7-Trimethylbicyclo[2.2.1] heptan-2-d-2ol (S4): Following the general procedure III to afford S4 (71.2mg, 46\% yield) as white solid (eluent: $V$ (hexane/ $V($ EtOAc $)=45 / 1)$. m.p. $217.2{ }^{\circ} \mathrm{C}$; ${ }^{1} \mathrm{H}$ NMR $(400 \mathrm{MHz}$, $\left.\mathrm{CDCl}_{3}\right) \delta: 1.71(\mathrm{p}, J=3.7 \mathrm{~Hz}, 3 \mathrm{H}), 1.68 \sim 1.61(\mathrm{~m}, 2 \mathrm{H})$, $1.52 \sim 1.44(\mathrm{~m}, 1 \mathrm{H}), 1.00(\mathrm{~d}, J=4.8 \mathrm{~Hz}, 4 \mathrm{H}), 0.97(\mathrm{~s}, 1 \mathrm{H})$, 0.89 (s, 3H), $0.81(\mathrm{~s}, 3 \mathrm{H}) ;{ }^{13} \mathrm{C}$ NMR $\left(101 \mathrm{MHz}, \mathrm{CDCl}_{3}\right) \delta$ : $79.6(\mathrm{t}, J=22.6 \mathrm{~Hz}), 49.0,46.5,45.2,40.4,34.0,27.4$, 20.6, 20.2, 11.4; IR (neat) $v: 3421,2950,2873,1636$, $1453,1078 \mathrm{~cm}^{-1}$. HRMS (ESI) calcd for $\mathrm{C}_{10} \mathrm{H}_{17} \mathrm{DONa}$ $[\mathrm{M}+\mathrm{Na}]^{+}:$178.1318, found 178.1311 .

2-((4-Phenylcyclohexyl-1-d)oxy)pyridine (7a): Following the general procedure I to afford 7a (41 mg, $81 \%$ yield) as white solid (eluent: $V$ (hexane) $\left./ V\left(\mathrm{Et}_{2} \mathrm{O}\right)=30 / 1\right)$. m.p. $111.1{ }^{\circ} \mathrm{C} ;{ }^{1} \mathrm{H}$ NMR $\left(400 \mathrm{MHz}, \mathrm{CDCl}_{3}\right) \delta: 8.19(\mathrm{~d}, J=$ $4.8 \mathrm{~Hz}, 1 \mathrm{H}), 7.58(\mathrm{t}, J=7.7 \mathrm{~Hz}, 1 \mathrm{H}), 7.34(\mathrm{t}, J=7.2 \mathrm{~Hz}$, $2 \mathrm{H}), 7.28 \sim 7.21(\mathrm{~m}, 3 \mathrm{H}), 6.86(\mathrm{t}, J=5.9 \mathrm{~Hz}, 1 \mathrm{H}), 6.74(\mathrm{~d}$, $J=8.3 \mathrm{~Hz}, 1 \mathrm{H}), 2.62(\mathrm{t}, J=11.7 \mathrm{~Hz}, 1 \mathrm{H}), 2.33(\mathrm{~d}, J=11.7$ $\mathrm{Hz}, 2 \mathrm{H}), 2.03$ (d, $J=12.6 \mathrm{~Hz}, 2 \mathrm{H}), 1.72$ (t, $J=13.1 \mathrm{~Hz}$, $3 \mathrm{H}), 1.63(\mathrm{t}, J=12.6 \mathrm{~Hz}, 2 \mathrm{H}) ;{ }^{13} \mathrm{C} \mathrm{NMR}(101 \mathrm{MHz}$, $\left.\mathrm{CDCl}_{3}\right) \delta: 163.6,147.0,146.8,138.7,128.5,126.9,126.2$, 116.5, 111.8, $73.0(\mathrm{t}, J=22.1 \mathrm{~Hz}), 43.8,32.6,32.4$; IR (neat) $v: 3027,2934,2853,1951,1567,1469,1307,1289$, $1075,785,754,700 \mathrm{~cm}^{-1}$. HRMS (ESI) calcd for $\mathrm{C}_{17} \mathrm{H}_{18^{-}}$ DNONa [M+Na] $]^{+}: 277.1427$, found 277.1420.

5-Chloro-2-((4-phenylcyclohexyl-1-d)oxy)pyridine (7b): Following the general procedure I to afford $7 \mathbf{b}(47.8 \mathrm{mg}$, $83 \%$ yield) as yellow solid (eluent: $V($ hexane $) / V\left(\mathrm{Et}_{2} \mathrm{O}\right)=$ 30/1). m.p. $80.9{ }^{\circ} \mathrm{C} ;{ }^{1} \mathrm{H}$ NMR (400 MHz, $\mathrm{CDCl}_{3}$ ) $\delta: 8.11$ (s, 1H), $7.53(\mathrm{~d}, J=8.8 \mathrm{~Hz}, 1 \mathrm{H}), 7.33(\mathrm{t}, J=7.2 \mathrm{~Hz}, 2 \mathrm{H})$, $7.28 \sim 7.22(\mathrm{~m}, 3 \mathrm{H}), 6.68(\mathrm{~d}, J=8.7 \mathrm{~Hz}, 1 \mathrm{H}), 2.60(\mathrm{t}, J=$ $11.6 \mathrm{~Hz}, 1 \mathrm{H}), 2.29(\mathrm{~d}, J=11.5 \mathrm{~Hz}, 2 \mathrm{H}), 2.01(\mathrm{~d}, J=12.5$ $\mathrm{Hz}, 2 \mathrm{H}), 1.73 \sim 1.64(\mathrm{~m}, 2 \mathrm{H}), 1.61(\mathrm{~d}, J=13.2 \mathrm{~Hz}, 2 \mathrm{H})$; ${ }^{13} \mathrm{C} \mathrm{NMR}\left(101 \mathrm{MHz}, \mathrm{CDCl}_{3}\right) \delta: 162.0,146.6,145.2,138.6$, $128.6,126.9,126.3,123.7,112.8,73.9(\mathrm{t}, J=22.0 \mathrm{~Hz})$, 43.7, 32.5, 32.2; IR (neat) $v$ : 3026, 2935, 2858, 1591, $1560,1463,1300,1283,1073,829,753,697 \mathrm{~cm}^{-1}$. HRMS (ESI) calcd for $\mathrm{C}_{17} \mathrm{H}_{17} \mathrm{DCINONa}\left[\mathrm{M}+\mathrm{Na}^{+}\right]$: 311.1037 , found 311.1031 .

4-Methyl-2-((4-phenylcyclohexyl-1-d)oxy)pyridine (7c): Following the general procedure I to afford 7c $(40.2 \mathrm{mg}$, $75 \%$ yield) as yellow solid (eluent: $V$ (hexane) $/ V\left(\mathrm{Et}_{2} \mathrm{O}\right)=$ 35/1). m.p. $81.5{ }^{\circ} \mathrm{C} ;{ }^{1} \mathrm{H}$ NMR (400 MHz, Chloroform- $d$ ) $\delta$ : $8.03(\mathrm{~d}, J=5.2 \mathrm{~Hz}, 1 \mathrm{H}), 7.33(\mathrm{t}, J=7.5 \mathrm{~Hz}, 2 \mathrm{H}), 7.25(\mathrm{t}$, $J=9.0,7.6 \mathrm{~Hz}, 3 \mathrm{H}), 6.69(\mathrm{~d}, J=5.2 \mathrm{~Hz}, 1 \mathrm{H}), 6.55(\mathrm{~s}, 1 \mathrm{H})$, $2.60(\mathrm{t}, J=11.9 \mathrm{~Hz}, 1 \mathrm{H}), 2.30(\mathrm{~d}, J=7.7 \mathrm{~Hz}, 5 \mathrm{H}), 2.01$ (d, $J=12.9 \mathrm{~Hz}, 2 \mathrm{H}), 1.70(\mathrm{t}, J=13.1 \mathrm{~Hz}, 2 \mathrm{H}), 1.60(\mathrm{t}, J=12.5$ $\mathrm{Hz}, 2 \mathrm{H}) ;{ }^{13} \mathrm{C} \mathrm{NMR}\left(101 \mathrm{MHz}, \mathrm{CDCl}_{3}\right) \delta: 163.9,150.0$, $146.8,146.5,128.5,126.9,126.2,118.1,111.9,72.9(\mathrm{t}, J=$ $22.1 \mathrm{~Hz}$ ), 43.8, 32.6, 32.4, 21.0; IR (neat) $v: 3025,2929$, 2856, 1610, 1560, 1402, 1317, 1161, 1070, 810, 755, 698 $\mathrm{cm}^{-1}$. HRMS (ESI) calcd for $\mathrm{C}_{18} \mathrm{H}_{20} \mathrm{DNONa}[\mathrm{M}+\mathrm{Na}]^{+}$: 291.1584, found 291.1576.

2-((4-Phenylcyclohexyl-1-d)oxy)pyrazine (7d): Following the general procedure I to afford $7 \mathbf{d}(36.7 \mathrm{mg}, 72 \%$ yield) as white solid (eluent: $V($ hexane $\left.) / V\left(\mathrm{Et}_{2} \mathrm{O}\right)=30 / 1\right)$. m.p.124.1 ${ }^{\circ} \mathrm{C} ;{ }^{1} \mathrm{H}$ NMR (400 MHz, $\left.\mathrm{CDCl}_{3}\right) \delta: 8.23$ (s, 1H), $8.11(\mathrm{~d}, J=6.4 \mathrm{~Hz}, 2 \mathrm{H}), 7.35$ (t, $J=7.2 \mathrm{~Hz}, 2 \mathrm{H}), 7.26(\mathrm{t}$, $J=7.4 \mathrm{~Hz}, 3 \mathrm{H}), 2.63(\mathrm{t}, J=10.0 \mathrm{~Hz}, 1 \mathrm{H}), 2.32$ (d, $J=10.3$ $\mathrm{Hz}, 2 \mathrm{H}), 2.05$ (d, $J=12.5 \mathrm{~Hz}, 2 \mathrm{H}), 1.74(\mathrm{t}, J=13.1 \mathrm{~Hz}$, $3 \mathrm{H}), 1.63 \sim 1.77(\mathrm{~m}, 4 \mathrm{H}) ;{ }^{13} \mathrm{C}$ NMR $\left(101 \mathrm{MHz}, \mathrm{CDCl}_{3}\right) \delta$ : $160.0,146.4,140.6,136.6,136.3,128.5,126.9,126.3,74.0$ (t, $J=22.2 \mathrm{~Hz}), 43.6,32.4,32.0$; IR (neat) $v: 3039,2941$, 2925, 2852, 1585, 1528, 1410, 1299, 1073, 849, 749, 702 $\mathrm{cm}^{-1}$. HRMS (ESI) calcd for $\mathrm{C}_{16} \mathrm{H}_{17} \mathrm{DN}_{2} \mathrm{ONa}[\mathrm{M}+\mathrm{Na}]^{+}$: 278.1380 , found 278.1372 .

2-(1,3-Dioxolan-2-yl)-6-((4-phenylcyclohexyl-1-d)oxy)pyridine (7e): Following the general procedure $\mathbf{I}$ to afford 7e $(33.7 \mathrm{mg}, 52 \%$ yield) as white solid (eluent: $V$ (hexane)/ $\left.V\left(\mathrm{Et}_{2} \mathrm{O}\right)=25 / 1\right) .{ }^{1} \mathrm{H} \mathrm{NMR}\left(400 \mathrm{MHz}, \mathrm{CDCl}_{3}\right) \delta: 7.61(\mathrm{t}$, $J=7.7 \mathrm{~Hz}, 1 \mathrm{H}), 7.34(\mathrm{t}, J=7.3 \mathrm{~Hz}, 2 \mathrm{H}), 7.30 \sim 7.20(\mathrm{~m}$, $3 \mathrm{H}), 7.09$ (d, $J=7.2 \mathrm{~Hz}, 1 \mathrm{H}), 6.72(\mathrm{~d}, J=8.3 \mathrm{~Hz}, 1 \mathrm{H}), 5.82$ (s, 1H), 4.23 (d, $J=6.6 \mathrm{~Hz}, 2 \mathrm{H}), 4.11(\mathrm{~d}, J=5.4 \mathrm{~Hz}, 2 \mathrm{H})$, $2.61(\mathrm{t}, J=11.4 \mathrm{~Hz}, 1 \mathrm{H}), 2.34(\mathrm{~d}, J=11.4 \mathrm{~Hz}, 2 \mathrm{H}), 2.02$ $(\mathrm{d}, J=12.6 \mathrm{~Hz}, 2 \mathrm{H}), 1.76 \sim 1.67(\mathrm{~m}, 2 \mathrm{H}), 1.65 \sim 1.56(\mathrm{~m}$, $2 \mathrm{H}) ;{ }^{13} \mathrm{C}$ NMR $\left(101 \mathrm{MHz}, \mathrm{CDCl}_{3}\right) \delta: 163.3,155.0,146.8$, $139.0,128.5,126.9,126.2,112.9,112.0,103.9,73.2(\mathrm{t}, J=$ $22.1 \mathrm{~Hz}$ ), 65.7, 43.8, 32.5, 32.2; IR (neat) $v: 3025,2928$, 2855, 1598, 1575, 1321, 1075, 796, $700 \mathrm{~cm}^{-1}$. m.p. $94.5{ }^{\circ} \mathrm{C}$; HRMS (ESI) calcd for $\mathrm{C}_{20} \mathrm{H}_{22} \mathrm{DNO}_{3} \mathrm{Na}$ : $[\mathrm{M}+$ $\mathrm{Na}]^{+}:$349.1638, found 349.1629.

2-(sec-Butyl)-6-((4-phenylcyclohexyl-1-d)oxy)pyrazine (7f): Following the general procedure $\mathbf{I}$ to afford $7 \mathbf{f}$ (56.6 $\mathrm{mg}, 91 \%$ yield) as colorless liquid (eluent: $V$ (hexane)/ $\left.V\left(\mathrm{Et}_{2} \mathrm{O}\right)=35 / 1\right) .{ }^{1} \mathrm{H} \mathrm{NMR}\left(400 \mathrm{MHz}, \mathrm{CDCl}_{3}\right) \delta: 8.04(\mathrm{~s}$, $1 \mathrm{H}), 7.90(\mathrm{~s}, 1 \mathrm{H}), 7.32$ (t, $J=7.2 \mathrm{~Hz}, 2 \mathrm{H}), 7.23$ (dd, $J=$ 15.9, 7.6 Hz, 3H), $3.19(\mathrm{dt}, J=6.7 \mathrm{~Hz}, 1 \mathrm{H}), 2.62(\mathrm{t}, J=$ $10.3 \mathrm{~Hz}, 1 \mathrm{H}), 2.30$ (d, $J=10.4 \mathrm{~Hz}, 2 \mathrm{H}), 2.03$ (d, $J=12.8$ $\mathrm{Hz}, 2 \mathrm{H}), 1.84(\mathrm{dd}, J=13.9,7.1 \mathrm{~Hz}, 1 \mathrm{H}), 1.78 \sim 1.60(\mathrm{~m}$, $5 \mathrm{H}), 1.27(\mathrm{~d}, J=6.9 \mathrm{~Hz}, 3 \mathrm{H}), 0.88(\mathrm{t}, J=7.3 \mathrm{~Hz}, 3 \mathrm{H}) ;{ }^{13} \mathrm{C}$ NMR $\left(101 \mathrm{MHz}, \mathrm{CDCl}_{3}\right) \delta: 157.7,152.2,146.6,137.7$, 135.4, 128.5, 126.9, 126.3, $73.6(\mathrm{t}, J=22.1 \mathrm{~Hz}), 43.7,36.7$, $32.4,32.2,28.3,18.4,12.2,1.2$; IR (neat) $v: 3027,2961$, 2932, 2858, 1536, 1404, 1073, 840, 756, $699 \mathrm{~cm}^{-1}$. HRMS (ESI) calcd for $\mathrm{C}_{20} \mathrm{H}_{26} \mathrm{DN}_{2} \mathrm{O}[\mathrm{M}+\mathrm{H}]^{+}: 312.2186$, found 312.2181.

5-Chloro-2-((4'-propyl-[1,1'-bi(cyclohexan)]-4-yl-4-d)ox y)pyridine (7g): Following the general procedure I to afford $7 \mathrm{~g}$ (57.5 mg, $86 \%$ yield) as yellow solid (eluent: $V($ hexane $\left.) / V\left(\mathrm{Et}_{2} \mathrm{O}\right)=50 / 1\right)$. m.p. $102.5{ }^{\circ} \mathrm{C} ;{ }^{1} \mathrm{H}$ NMR $(400$ $\left.\mathrm{MHz}, \mathrm{CDCl}_{3}\right) \delta: 8.06(\mathrm{~s}, 1 \mathrm{H}), 7.48(\mathrm{~d}, J=8.8 \mathrm{~Hz}, 1 \mathrm{H})$, $6.62(\mathrm{~d}, J=8.8 \mathrm{~Hz}, 1 \mathrm{H}), 2.13(\mathrm{~d}, J=12.4 \mathrm{~Hz}, 2 \mathrm{H}), 1.80 \sim$ $1.69(\mathrm{~m}, 6 \mathrm{H}), 1.36 \sim 1.26(\mathrm{~m}, 4 \mathrm{H}), 1.18 \sim 1.06(\mathrm{~m}, 7 \mathrm{H})$, $0.99 \sim 0.93(\mathrm{~m}, 2 \mathrm{H}), 0.89 \sim 0.83(\mathrm{~m}, 5 \mathrm{H}) ;{ }^{13} \mathrm{C}$ NMR $(101$ $\left.\mathrm{MHz} \mathrm{CDCl}_{3}\right) \delta: 162.1,145.2,138.5,123.5,112.7,74.6(\mathrm{t}$, 
$J=22.2 \mathrm{~Hz}$ ), 43.0, 42.6, 39.9, 37.7, 33.7, 32.1, 30.3, 28.2, 20.2, 14.6; IR (neat) $v: 3021,2924,2856,1591,1466$, 1374, 1284, 1061, 823, $696 \mathrm{~cm}^{-1}$. HRMS (ESI) calcd for $\mathrm{C}_{20} \mathrm{H}_{29} \mathrm{DCINONa}[\mathrm{M}+\mathrm{Na}]^{+}: 359.1976$, found 359.1974.

4-((4'-Propyl-[1,1'-bi(cyclohexan)]-4-yl-4-d)oxy)quinoline (7h): Following the general procedure $\mathbf{I}$ to afford $\mathbf{7 h}$ (48.4 mg, 69\% yield) as white solid (eluent: $V$ (hexane/ $V($ EtOAc $)=25 / 1) .{ }^{1} \mathrm{H}$ NMR $\left(400 \mathrm{MHz}, \mathrm{CDCl}_{3}\right) \delta: 8.70(\mathrm{~d}$, $J=5.2 \mathrm{~Hz}, 1 \mathrm{H}), 8.20(\mathrm{~d}, J=8.3 \mathrm{~Hz}, 1 \mathrm{H}), 8.00(\mathrm{~d}, J=8.4$ $\mathrm{Hz}, 1 \mathrm{H}), 7.66$ (t, $J=7.6 \mathrm{~Hz}, 1 \mathrm{H}), 7.46$ (t, $J=7.5 \mathrm{~Hz}, 1 \mathrm{H})$, $6.71(\mathrm{~d}, J=5.3 \mathrm{~Hz}, 1 \mathrm{H}), 2.26$ (d, $J=11.8 \mathrm{~Hz}, 2 \mathrm{H}), 1.88$ (d, $J=5.7 \mathrm{~Hz}, 2 \mathrm{H}), 1.75(\mathrm{t}, J=13.2 \mathrm{~Hz}, 4 \mathrm{H}), 1.54$ (t, $J=10.7$ $\mathrm{Hz}, 2 \mathrm{H}), 1.36 \sim 1.26(\mathrm{~m}, 4 \mathrm{H}), 1.21 \sim 1.12(\mathrm{~m}, 6 \mathrm{H}), 1.07(\mathrm{~d}$, $J=12.4 \mathrm{~Hz}, 1 \mathrm{H}), 0.99$ (d, $J=12.4 \mathrm{~Hz}, 2 \mathrm{H}), 0.88$ (t, $J=7.3$ $\mathrm{Hz}, 5 \mathrm{H}) ;{ }^{13} \mathrm{C}$ NMR $\left(101 \mathrm{MHz}, \mathrm{CDCl}_{3}\right) \delta: 160.8,151.3$, $149.6,129.7,128.9,125.4,122.3,101.4,76.9$ (t, $J=21.2$ Hz), 42.8, 42.5, 39.9, 37.7, 33.6, 31.8 30.3, 28.0, 20.2, 14.5; IR (neat) v: 3058, 2948, 2846, 1585, 1503, 1393, $1310,1110,817,770 \mathrm{~cm}^{-1}$. HRMS (ESI) calcd for $\mathrm{C}_{24} \mathrm{H}_{33^{-}}$ DNO $[\mathrm{M}+\mathrm{H}]^{+}: 353.2703$, found 353.2694 .

2-((Cyclooctyl-1-d)oxy)pyridine (7i): Following the general procedure I to afford $7 \mathbf{i}(42.3 \mathrm{mg}, 96 \%$ yield) as colorless liquid (eluent: $V$ (hexane) $\left./ V\left(\mathrm{Et}_{2} \mathrm{O}\right)=80 / 1\right) .{ }^{1} \mathrm{H}$ NMR (400 MHz, $\left.\mathrm{CDCl}_{3}\right) \delta: 8.13(\mathrm{~d}, J=4.6 \mathrm{~Hz}, 1 \mathrm{H}), 7.51$ $(\mathrm{t}, J=7.1 \mathrm{~Hz}, 1 \mathrm{H}), 6.78(\mathrm{t}, J=6.0 \mathrm{~Hz}, 1 \mathrm{H}), 6.65(\mathrm{~d}, J=8.3$ $\mathrm{Hz}, 1 \mathrm{H}), 1.96 \sim 1.90(\mathrm{~m}, 2 \mathrm{H}), 1.88 \sim 1.80(\mathrm{~m}, 2 \mathrm{H}), 1.79 \sim$ $1.70(\mathrm{~m}, 2 \mathrm{H}), 1.60(\mathrm{~s}, 8 \mathrm{H}) ;{ }^{13} \mathrm{C} \mathrm{NMR}\left(101 \mathrm{MHz}, \mathrm{CDCl}_{3}\right) \delta$ : 163.6, 147.1, 138.5, 116.2, 111.8, 74.9 (t, $J=22.3 \mathrm{~Hz}$ ), $31.5,27.5,25.6,23.1$; IR (neat) $v: 3014,2923,2853,1593$, 1469, 1429, 1310, 1289, 1059, $777 \mathrm{~cm}^{-1}$. HRMS (ESI) calcd for $\mathrm{C}_{13} \mathrm{H}_{19} \mathrm{DNO}[\mathrm{M}+\mathrm{H}]^{+}$: 207.1601, found 207.1600 .

5-Chloro-2-(((1S,2R,4S)-1,7,7-trimethylbicyclo[2.2.1]heptan-2-yl-2-d)oxy)pyridine (7j): Following the general procedure I to afford $7 \mathbf{j}$ (48.1 $\mathrm{mg}, 91 \%$ yield) as yellow liquid (eluent: $V($ hexane $\left.) / V\left(\mathrm{Et}_{2} \mathrm{O}\right)=50 / 1\right)$. m.p. $80.9{ }^{\circ} \mathrm{C}$; ${ }^{1} \mathrm{H}$ NMR $\left(400 \mathrm{MHz}, \mathrm{CDCl}_{3}\right) \delta: 8.06(\mathrm{~s}, 1 \mathrm{H}), 7.47(\mathrm{~d}, J=$ $8.8,2.5 \mathrm{~Hz}, 1 \mathrm{H}), 6.62$ (d, $J=8.8 \mathrm{~Hz}, 1 \mathrm{H}), 1.90$ (d, $J=12.9$ $\mathrm{Hz}, 1 \mathrm{H}), 1.76 \sim 1.71(\mathrm{~m}, 2 \mathrm{H}), 1.58(\mathrm{t}, J=11.8 \mathrm{~Hz}, 1 \mathrm{H})$, $1.43(\mathrm{~s}, 1 \mathrm{H}), 1.21(\mathrm{t}, J=12.5 \mathrm{~Hz}, 1 \mathrm{H}), 1.12(\mathrm{t}, J=10.0 \mathrm{~Hz}$, $1 \mathrm{H}), 1.06(\mathrm{~s}, 3 \mathrm{H}), 0.91(\mathrm{~s}, 3 \mathrm{H}), 0.86(\mathrm{~s}, 3 \mathrm{H}) ;{ }^{13} \mathrm{C} \mathrm{NMR}$ $\left(101 \mathrm{MHz}, \mathrm{CDCl}_{3}\right) \delta: 162.3,145.2,138.4,123.5,112.6$, $82.5(\mathrm{t}, J=23.7 \mathrm{~Hz}), 48.9,47.3,45.3,39.4,34.1,30.5$, 27.3, 20.4, 20.2, 11.9; IR (neat) $v: 3009,2953,2876,1590$, 1463, 1375, 1289, 1074, 824, $702 \mathrm{~cm}^{-1}$. HRMS (ESI) calcd for $\mathrm{C}_{15} \mathrm{H}_{19} \mathrm{DClNONa}[\mathrm{M}+\mathrm{Na}]^{+}: 289.1194$, found 289.1186.

Supporting Information The ${ }^{1} \mathrm{H}$ NMR and ${ }^{13} \mathrm{C}$ NMR spectra of $\mathbf{2 a}, \mathbf{2 d}, \mathbf{4 a} \sim \mathbf{4 z}, \mathbf{4 a a}, \mathbf{7 a} \sim \mathbf{7 j}$ and $\mathbf{S 1} \sim \mathbf{S 4}$. The Supporting Information is available free of charge via the Internet at http://sioc-journal.cn/.

\section{References}

[1] (a) Buckingham, J. Dictionary of Natural Products, University Press, Cambridge, MA, 1994.

(b) Evano, G.; Wang, J.; Nitelet, A. Org. Chem. Front. 2017, 4,
2480.

(c) Enthaler, S.; Company, A. Chem. Soc. Rev. 2011, 40, 4912.

(d) Theil, F. Angew. Chem. Int. Ed. 1999, 38, 2345.

(e) Muci, A. R.; Buchwald, S. L. Top. Curr. Chem. 2002, 219, 131.

(f) Thomas, A. W.; Ley, S. V. Angew. Chem., Int. Ed. 2003, 42, 5400 .

[2] (a) Manchado, E.; Weissmueller, S.; Morris, J. P.; Chen, C. C.; Wullenkord, R.; Lujambio, A.; Stanchina, E.; Poirier, J. T.; Gainor, J. F.; Corcoran, R. B.; Engelman, J. A.; Rudin, C. M.; Rosen, N.; Lowe, S. W. Nature 2016, 534, 647.

(b) Huang, S.; Hölzel, M.; Knijnenburg, T.; Schlicker, A.; Roepman, P.; McDermott, U.; Garnett, M.; Grernrum, W.; Sun, C.; Prahallad, A.; Groenendijk, F. H.; Mittempergher, L.; Nijkamp, W.; Neefjes, J.; Salazar, R.; Dijke, P.; Uramoto, H.; Tanaka, F.; Beijersbergen, R. L.; Wessels, L. F.; Bernards, R. Cell 2012, 151, 937. (c) Wang, S.; Cang, S.; Liu, D. J. Hematol. Oncol. 2016, 9, 34.

[3] (a) Muci, A. R.; Buchwald, S. L. Practical Palladium Catalysts for $\mathrm{C}-\mathrm{N}$ and $\mathrm{C}-\mathrm{O}$ Bond Formation. In Topics in Current Chemistry, Ed.: Miyaura, N. E., Springer-Verlag, Berlin, Germany, 2001, Vol. 219, p. 131

(b) Hartwig, J. F. In Handbook of Organopalladium Chemistry for Organic Synthesis, Ed.: Negishi, E. E., Wiley-Interscience, New York, 2002, p. 1051.

(c) Beletskaya, I. P.; Cheprakov, A. V. Coord. Chem. Rev. 2004, 248, 2337.

(d) Bedford, R. B.; Cazin, C. S. J.; Holder, D. Coord. Chem. Rev. 2004, 248, 2283.

(e) Beccalli, E. M.; Broggini, G.; Martinelli, M.; Sottocornola, S. Chem. Rev. 2007, 107, 5318.

[4] (a) Vorogushin, A. V.; Huang, X.; Buchwald, S. L. J. Am. Chem. Soc. 2005, 127, 8146 .

(b) Zhang, H.; Ruiz-Castillo, P.; Buchwald, S. L. Org. Lett. 2018, 20, 1580 .

[5] Chen, Z.; Jiang, Y.; Zhang, L.; Guo, Y.; Ma, D. J. Am. Chem. Soc. 2019, 141, 3541 .

[6] Terrett, J. A.; Cuthbertson, J. D.; Shurtleff, V. W.; MacMillan, D. W. C. Nature 2015, 524, 330.

[7] (a) Williamson, A. Philos. Mag. 1850, 37, 299.

(b) Fuhrmann, E.; Talbiersky, J. Org. Process Res. Dev. 2005, 9, 206.

[8] Swamy, K. C. K.; Kumar, N. N. B.; Balaraman, E.; Kumar, K. V. P. P. Chem. Rev. 2009, 109, 2551.

[9] Caron, S.; Ghosh, A. Nucleophilic Aromatic Substitution. In Practical Synthetic Organic Chemistry Reactions Principles and Techniques, John Wiley \& Sons, Hoboken, NJ, 2011, p. 237.

[10] For representative publications:

(a) Zarate, C.; Manzano, R.; Martin, R. J. Am. Chem. Soc. 2015, 137, 6754.

(b) Zarate, C.; Nakajima, M.; Martin, R. J. Am. Chem. Soc. 2017, 139, 1191 .

(c) Sergeev, A. G.; Hartwig, J. F. Science 2011, 332, 439.

(d) Álvarez-Bercedo, P.; Martin, R. J. Am. Chem. Soc. 2010, 132, 17352.

(e) Guan, B.; Xiang, S.; Wang, B.; Sun, Z.; Wang, Y.; Zhao, K.; Shi, Z. J. Am. Chem. Soc. 2008, 130, 3268.

(f) Guo, L.; Liu, X.; Baumann, C.; Rueping, M. Angew. Chem. Int. Ed. 2016, 55, 15415.

(g) Liu, X.; Hsiao, C. C.; Kalvet, I.; Leiendecker, M.; Guo, L.; Schoenebeck, F.; Rueping, M. Angew. Chem. Int. Ed. 2016, 55, 6093.

(h) Cong, X.; Tang, H.; Zeng, X. J. Am. Chem. Soc. 2015, 137, 14367.

(i) Tobisu, M.; Takahira, T.; Morioka, T.; Chatani, N. J. Am. Chem. Soc. 2016, 138, 6711.

(j) Wang, X.; Li, C.; Wang, X.; Wang, Q.; Dong, X.; Duan, A.; Zhao, W. Org. Lett. 2018, 20, 4267.

(k) Wang, X.; Tang, Y.; Long, C.; Dong, W.; Li, C.; Xu, X.; Zhao, W.; Wang, X. Org. Lett. 2018, 20, 4749. 
(1) Tang, N.; Shao, X.; Wang, M.; Wu, X.; Zhu, C. Acta Chim. Sinica 2019, 77, 922.

(m) Huang, S.; Nie, Y.; Yang, J.; Zheng, Z.; Cao, J.; Xu, Z.; Xu, L. Chin. J. Org. Chem. 2020, 40, 2018.

(n) Tao, X.; Sheng, R.; Bao, K.; Wang, Y.; Jin, Y. Chin. J. Org. Chem. 2019, 39, 2726.

[11] (a) Kwan, E. E.; Zeng, Y.; Besser, H. A.; Jacobsen, E. N. Nat. Chem. 2018, 10, 917.

(b) Neumann, C. N.; Ritter, T. Acc. Chem. Res. 2017, 50, 2822.

(c) Terrier, F. Modern Nucleophilic Aromatic Substitution, Wiley-VCH Verlag GmbH \& Co. KGaA, Weinheim, Germany, 2013.

[12] Representative examples for quinoline containing ether type anticancer drugs:

(a) Xie, C.; Wan, X.; Quan, H.; Zheng, M.; Fu, L.; Li, Y.; Lou, L. Cancer Sci. 2018, 109, 1207.

(b) Wang, T.; Xiao, M.; Ge, Y.; Krepler, C.; Belser, E.; Lopez-Coral, A.; Xu, X.; Zhang, G.; Azuma, R.; Liu, Q.; Liu, R.; Li, L.; Amara- vadi, R. K.; Xu, W.; Karakousis, G.; T. Gangadhar, C.; Schuchter, L. M.; Lieu, M.; Khare, S.; Halloran, M. B.; Herlyn, M.; Kaufman, R. E. Clin. Cancer Res. 2015, 21, 1652.

[13] Li, F.; Fernandez, P. P.; Rajendran, P.; Hui, K. M.; Sethi, G. Cancer Lett. 2010, 292, 197.

[14] (a) DeVore, N. M.; Scott, E. E. Nature 2012, 482, 116.

(b) Abedinpour, P.; Baron, V. T.; Chrastina, A.; Rondeau, G.; Pelayo, J.; Welsh, J.; Borgström, P. Prostate 2017, 77, 1550.

[15] Richtand, N. M.; Welge, J. A.; Logue, A. D.; Keck, P. E.; Strakowski, S. M.; McNamara, R. K. Neuropsychopharmacology. 2007, 32, 1715.

[16] (a) Atzrodt, J.; Derdau, V.; Kerr, W. J.; Reid, M. Angew. Chem., Int. Ed. 2018, 57, 1758.

(b) Gant, T. G. J. Med. Chem. 2014, 57, 3595.

(c) Pirali, T.; Serafini, M.; Cargnin, S.; Genazzani, A. A. J. Med. Chem. 2019, 62, 5276 .

(d) Chang, Y.; Yesilcimen, A.; Cao, M.; Zhang, Y.; Zhang, B.; Chan, J. Z.; Wasa, M. J. Am. Chem. Soc. 2019, 141, 14570.

(Fan, Y.) 